\title{
„PER L'ALLUSIONE ALLE CORRENTI COSE D'ITALIA“" ANTONIO CALDARAS RÖMISCHE WEIHNACHTSKANTATEN FÜR PAPST UND FÜRST
}

\section{Einleitung}

In den Commentari zu seiner L'Istoria della volgar Poesia geht der römische Arkadier Giovanni Mario Crescimbeni an einer Stelle gezielt auf die Texte jener Kompositionen ein, die um 1700 anlässlich der Weihnachtsfeierlichkeiten am Papsthof erklungen waren. Crescimbenis Anmerkungen zu einem Kantatentext von Francesco Maria Campello sind dabei ein wichtiger Schlüssel zum Verständnis der zeitgenössischen Wahrnehmung jener Werke und sollen Ausgangspunkt für die Untersuchungen der Weihnachtskantaten sein, die von Antonio Caldara in Musik gesetzt wurden. Im Falle Campellos legt Crescimbeni dar, der Textdichter habe nicht allein für die „dolcezza“ des Verses und für die Vortrefflichkeit des Feingefühls, sondern gleichermaßen für die Anspielung auf die „,correnti cose d'Italia" im Jahr 1701 große Hochachtung erhalten.

Auch in den Texten jener Kantaten, die Caldara während seiner Dienstzeit (1709-1716) bei dem römischen Adeligen Francesco Maria Ruspoli für verschiedene Weihnachtsfeierlichkeiten vertonte, spielen die „,correnti cose d'Italia“ eine zentrale Rolle. Die Entstehung der drei erhaltenen Werke (Vaticini di pace, Amarilli vezzosa ${ }^{2}$ und die Cantata da recitarsi la notte del SS. ${ }^{\text {mo }}$ Natale, Vo 'piangendo e sospirando) fällt nämlich exakt in jene Zeit, die von den Friedensverhandlungen zur Beendigung des Spanischen Erbfolgekrieges geprägt war. Hatte die Eröffnung der Friedensberatungen in Utrecht im Januar 1712 für Papst Clemens XI. unter schlechten Vorzeichen gestanden, so offenbarten die Friedensgespräche doch zweierlei: Zum einen hatte im Laufe des Krieges die machtpolitische Rolle

1 CRESCIMBENI, Giovanni Mario. L'Istoria della volgar Poesia scritta da Gio. Mario Crescimbeni. Canonico di Santa Maria in Cosmedin, e Custode d'Arcadia. Nella Seconda Impressione. Venedig: Lorenzo Basegio, 1731, S. 314. 
des Pontifex, zum anderen auch sein Image als politischer Mediator erheblichen Schaden genommen. Militärisch hatte das Oberhaupt des Kirchenstaates gegen das kaiserliche Heer im Comacchio-Krieg (1708/09) nicht reüssieren können und seine - von kaiserlicher Seite immer wieder angeprangerte - profranzösische Haltung diskreditierte ihn nun als politisch neutralen (Friedens-)Vermittler. ${ }^{3}$ Die Ewige Stadt selbst war zwar nicht unmittelbar vom Kriegsgeschehen betroffen gewesen, doch war sie während der Kämpfe zum „Hauptschlachtfeld der Propaganda" geworden, eine Propaganda, ,die sich nicht nur schriftlicher und bildlicher, sondern auch musikalischer Mittel bediente". ${ }^{4}$ Saverio Franchi stützt diesen Befund, indem er bereits zuvor nachgewiesen hatte, dass sich eine politisch beeinflusste inhaltliche Prägung einer großen Anzahl von Oratorien, Serenaten und Kantaten konstatieren lasse, die zu jener Zeit in Rom entstanden waren. ${ }^{5}$ Dies gilt gleichermaßen für die weihnachtlichen Kompositionen Caldaras. Bereits 1964 hatte Ursula Kirkendale den Einfluss der zeitgenössischen Kriegshandlungen im Werk des Komponisten thematisiert, fokussierte in ihrem Artikel jedoch Werke für den Mantuaner Herzog Ferdinando Carlo di Gonzaga-Nevers. ${ }^{6}$

$\mathrm{Ob}$ und in welcher Form Caldaras römische Weihnachtskantaten von den politischen Umständen beeinflusst wurden, wird im folgenden Beitrag zu klären sein. Dabei wird eingangs untersucht, für welche Auftraggeber und Darbietungskontexte diese Werke entstanden sind und wie der soziokulturelle bzw. politische Kontext die Kantaten prägte. In diesem Zusammenhang werden zudem Datierungsprobleme der Kompositionen behandelt. ${ }^{7}$ Darauf aufbauend gilt es, die textlichen wie musikalischen Zusammenhänge innerhalb der betrachteten Werke darzulegen. Zum Abschluss wird der Frage nachgegangen, ob jene Kompositionen als Teil der römischen Propaganda zu betrachten sind, die den Papst im Zuge der Friedensverhandlungen zum Spanischen Erbfolgekrieg als Friedensstifter stilisierten. $^{8}$

3 Vgl. zur Rolle des Papstes und zu den Schwierigkeiten der päpstlichen Neutralität während des Spanischen Erbfolgekrieges: TABACCHI, Stefano. L'impossibile neutralità. Il papato, Roma e lo Stato della Chiesa durante la Guerra di Successione spagnola. In Famiglie, nazioni e Monarchia. Il sistema europeo durante la Guerra di Successione spagnola, hrsg. von Antonio Álvarez-Ossorio Alvariño. Rom: Bulzoni, 2004, S. 223-244.

4 SCHNETTGER, Matthias. Der Spanische Erbfolgekrieg. 1701-1713/14. München: C. H. Beck, 2014, S. 60.

5 Vgl. FRANCHI, Saverio. Mecenatismo musicale e poesia per musica a Roma nei primi decenni dell'Arcadia. In Atti e memorie dell'Arcadia, 2012, 1, S. 81-116.

6 Vgl. KIRKENDALE, Ursula. The War of the Spanish Succession Reflected in Works of Antonio Caldara. In Acta Musicologica, 1964, 36/4, S. 221-233.

7 Vgl. zum untersuchten Quellenmaterial die Tabelle im Anhang 1, die über Titel, Textautor, Besetzung sowie Datierung der jeweiligen Kantate informiert.

8 Vgl. zu den Ausprägungen der Friedenskultur die maßgebliche Publikation: DURCHHARDT, Heinz und ESPENHORST, Martin (Hrsg.). Utrecht - Rastatt - Baden 1712-1714. Ein europäisches Friedenswerk am Ende des Zeitalters Ludwig XIV. Göttingen: Vandenhoeck \& Ruprecht, 2013. 


\section{Anlass, Auftraggeber und Aufführungskontext der Weihnachtskantaten}

\subsection{Caldaras Kantatenkomposition für die Weihnachtsfeierlichkeiten am Papsthof}

Seit dem Jahr 1676 lässt sich eine musikalische Tradition am Papsthof nachweisen, die vorsah, an Weihnachten nach den Primi Vespri di Natale eine Kantate oder ein Oratorium von der Cappella pontificia vortragen zu lassen. So entstand bis $1740^{9}$ ein umfassendes Repertoire von Werken für die Weihnachtsfeierlichkeiten im Palazzo Apostolico. Beredte Zeugnisse dieser Tradition sind die gedruckten Textbücher, die - im Gegensatz zu den Musikalien - beinahe lückenlos erhalten sind. ${ }^{10}$ Neben den Libretti sind es vor allem die zeitgenössischen Ausführungen von Andrea Adami, Matteo Fornari sowie von Francesco Cancellieri, ${ }^{11}$ die es erlauben, die Kantatenkompositionen einer spezifischen Funktion zuzuordnen, sie im Rahmen der Festlichkeiten zu verorten und zu kontextualisieren. Dies soll im Folgenden anhand Caldaras einziger für den Papsthof entstandener Cantata da recitarsi la notte del SS. ${ }^{m o} N a t a l e$, Vo 'piangendo e sospirando ${ }^{12}$ exemplifiziert werden.

Gemäß den Ausführungen Adamis ${ }^{13}$ ist davon auszugehen, dass Caldara vom päpstlichen Maggiordomo - in diesem Fall Fabio Abati Olivieri ${ }^{14}$ - mit der Auf-

9 Das Ende der Tradition scheint mit dem Tod Kardinal Pietro Ottobonis, der als Kardinalsprotektor der Cappella pontificia vorstand, eingetreten zu sein. Vgl. MARX, Hans Joachim. Römische Weihnachtsoratorien aus der ersten Hälfte des 18. Jahrhunderts. In Archiv für Musikwissenschaft, 1992, 49/3, S. 163-199, hier S. 169.

10 Vgl. zu den Textbüchern und den erhaltenen Partituren die Auflistungen bei ibid., S. 170-174. ders. „... da cantarsi nel Palazzo Apostolico“. Römische Weihnachtsoratorien des 17. Jahrhunderts. In Musikkulturgeschichte. Festschrift für Constantin Floros zum 60. Geburtstag, hrsg. v. Peter Petersen. Wiesbaden: Breitkopf \& Härtel, 1990, S. 415-424, hier S. 417-418; ebenso bei: GIANTURCO, Carolyn. ,Cantate Spirituali e Morali‘, with a Description of the Papal Sacred Cantata Tradition for Christmas 1676-1740. In Music \& Letters, 1992, 73/1, S. 1-31, hier S. 14-24.

11 Vgl. hierzu: ADAMI, Andrea. Osservazioni per ben regolare il coro dei Cantori della Cappella pontificia. Rom: Antonio de Rossi, 1711, S. 101; CANCELLIERI, Francesco. Descrizione de Tre Pontificali che si celebrano nella Basilica Vaticana per le Feste di Natale di Pasqua e di S. Pietro. Rom: Stamperia Vaticana, 1788, S. 19; FORNARI, Matteo. Istruzione Per gl'Officiali della Cappella Pontificia con diverse Osservazioni contenute nei Diarij de Puntatori Dall'Anno 1535 al 1736 a Tenore delle Costituzioni, Decreti, e Consuetudini della medesima Regnando la Santità di N.ro Sig.re Clemente Papa XII. Anno MDCCXXXVII. I-Rvat, Fondo Cappella Sistina 639, S. 97-99.

12 Vgl. zum Libretto: CALDARA, Antonio und GINI, Paolo. Cantata da recitarsi la notte del SS. ${ }^{\text {mo }}$ Natale nel Palazzo Apostolico l'anno MDCCXIII. Rom: Stamperia della Reverendissima Camera Apostolica, 1713, [cit. 2014-01-02]. URL: <http://daten.digitale-sammlungen. de/bsb00049909/image 1>. Vgl. zur Partitur Cantata à 3. Voci con Strom.ti|Per la Notte del SS.mo Natale | Del Sigr Antonio Caldara, GB-Lam MS 46.

ADAMI, op. cit., S. 102.

14 Vgl. hierzu auch: FRANCHI, Saverio. Il principe Ruspoli. L'oratorio in Arcadia. In Percorsi 
gabe betraut wurde, auf Grundlage eines Textes von Paolo Gini eine Cantata a tre voci für das Weihnachtsfest des Jahres 1713 zu verfassen. Vom Maestro di Cappella und dem Maggiordomo, der hauptverantwortlich für die Organisation der Aufführung des Werkes war, wurden überdies die Vokal- wie auch die Instrumentalbesetzung festgelegt. ${ }^{15}$ Die Musikalienkopie aus der Feder des römischen Kopisten Francesco Lanciani gibt außergewöhnlich detailreich über die Zusammensetzung des Ensembles Auskunft. Eine von fremder Hand hinzugefügte Notiz gibt an, dass neben drei Solisten 16 Sänger den Chor gestalteten. Das Concertino war mit zwei Violinen, einem Violoncello, einer Laute, einem Cembalo und einem Kontrabass besetzt; das Concerto grosso wies je zwölf erste und zweite Violinen, ergänzt von zwölf Bassi und sechs Violette, auf. ${ }^{16}$ Als Solisten seien - so Adami - die „migliori Cantori“"17 der Cappella auszuwählen, bei der Chorbesetzung gelte es laut Fornari nach dem Anciennitätsprinzip zu verfahren. ${ }^{18}$

dell'oratorio romano. Da „Historia Sacra“ a Melodramma Spirituale, hrsg. von dems. Rom: Ibimus, 2002, S. 245-316, hier: S. 302-303.

FORNARI, op. cit., S. 98.

16 „Numero dè Stromenti e Voci che v'intervennero nella sua rappresentazione che fù L'Anno MDCCXIII. Le Parole sono del Dottor Paolo Gini oltre Le trè voci Principali vi furono 16 altri Musici per Cantare il Coro ultimo. Per il Concertino 2. Violini, Violoncello, Leuto, Cembalo ed un Contrabasso. 12 primi e 12 secondi violini del Concerto Grosso. 12 Bassi, trà Violoni e Contrabassi, e 6 Violetti: in tutto 67 persone." GB-Lam MS 46.

17 ADAMI, op. cit., S. 102. Die Namen der Solisten konnten nicht ermittelt werden.

18 Vgl. FORNARI, op. cit., S. 98. Im Zusammenhang mit dem Gesangsensemble, das den Schlusschor zu gestalten hatte, ist ein Dokument von Interesse, das sich im Archiv der Familie Ruspoli erhalten hat. Es handelt sich hierbei um eine Abrechnung vom 31. Dezember 1713 ,per la cantata di Natale 1713“. (Die Abrechnung ist abgedruckt bei: KIRKENDALE, Ursula. Antonio Caldara. Life and Venetian-Roman Oratorios, 2. durchgesehene und übersetzte Aufl. Florenz: Leo S. Olschki Editore, 2007, S. 470.) Francesco Maria Ruspoli, Caldaras langjähriger Dienstherr in Rom, ließ via seinen Cameriere Pietro Castrucci vier Sänger, einen Violinisten und einen Violaspieler bezahlen. Auf Grundlage dieser Abrechnung nimmt Ursula Kirkendale an, dass Caldara eine „Christmas cantata $a$ 4“ (ibid. S. 89) für Ruspoli komponiert habe. Gegen diese Annahme spricht in erster Linie, dass sich weder eine Komposition für diese Sängerbesetzung noch eine Abrechnung für eine Kantatenkopie, wie sie für Ruspoli üblicherweise angefertigt wurde, ausmachen lässt. Die Aufführung einer größer besetzten Kantate ohne Beteiligung jener Solistinnen, die bei Ruspoli angestellt waren, wäre zudem außergewöhnlich gewesen. Ungewöhnlich erscheint auch das in der genannten Abrechnung verzeichnete Entgelt, da ohne Differenzierung zwischen Sängern und Musikern je ein Scudo ausbezahlt wurde. Im Regelfall wurden die Sänger mit einem höheren Betrag als die Streicher bedacht, es sei denn, sie wurden für die Ausführung eines Chores herangezogen, wie es beispielgebend bei der Bezahlung für die Aufführung der 16 Choristen des im Palazzo Ruspoli aufgeführten Oratorio per la Santissima Annunziata der Fall war. (Vgl. den Eintrag in der Lista dell'oratorio ultimo dell'Annunziata la sera della Domenica in Albis: „Per il coro de musici sedici importa S[cudi] 16“, ibid., S. 474.) Auf Basis dieser Überlegungen scheint es wahrscheinlich, dass es nicht zu einer weiteren Komposition einer Weihnachtskantate für die Casa Ruspoli gekommen war, sondern die genannten Musiker außertourlich dafür bezahlt wurden, um an der Cantata im Palazzo Apostolico mitzuwirken. Franchi identifizierte drei der vier aufgelisteten Sänger wie folgt: Pietro Ubaldo Sesti (Sopran), Giacomo Bonaventura Fei (Alt) und Giuseppe Antonio Guerrieri (Bass). Vgl. FRANCHI, 2002, op. cit., S. 303. 
Adamis Osservazioni geben neben den Informationen über die Verantwortlichkeit bei der Besetzung der „Cantata volgare sopar la Natività del Bambino Gesù “19 auch Auskunft über die Verankerung des Musikwerkes im Tagesablauf der Weihnachtsfeierlichkeiten am Papsthof. So war die musikalische Darbietung nicht mit einer liturgischen Feier, sondern mit einem besonders festlichen Abendessen verknüpft. Diese „lautissima Cena“ ${ }^{\text {“20 }}$ wurde jenen Kardinälen zuteil, die in der Nacht bei der Matutin und der Messe assistierten. Das Festmahl wurde von der Reverenda Camera Apostolica finanziert und mit besonderen Tischaufbauten (meist aus Zuckerwerk und Marzipan) gestaltet. Deren Aufmachung war thematisch vom Weihnachtsgedanken - genauer: vom Weihnachtsevangelium - geprägt. ${ }^{21}$ Musik und Abendessen bildeten eine Einheit und somit eine Zäsur innerhalb des von liturgischen Feiern geprägten Tagesablaufs am 24. Dezember. Im Falle der Feierlichkeiten von 1713, die nicht im Vatikan, sondern im Quirinalspalast stattfanden, wurde Caldaras Werk nach der Weihnachtsvesper „cantato da più celebri Musici accompagnato da virtuose Sinfonie“ in der Anticamera des Papstneffen Kardinal Annibale Albani vorgetragen. ${ }^{22}$ Im Anschluss an die Musik begleiteten

Kirkendale gibt Urbano Ferausa als Violinist und Bartolomeo Mossi als Violaspieler an. Vgl. KIRKENDALE, 2007, op. cit., S. 443.

ADAMI, op. cit., S. 102. Im Zusammenhang mit der Bezeichnung „Cantata“ darf die in der Literatur diskutierte Gattungsproblematik nicht unerwähnt bleiben: Gianturco spricht mit Blick auf das Repertoire von „Christmas Cantatas“ (GIANTURCO, op. cit.), während Marx (MARX, 1992, op. cit.) dieselben Werke als „Weihnachtsoratorien“" klassifiziert. Bis dato wurden die weihnachtlichen Vokalwerke für den Papsthof in ihrer Gesamtheit noch keiner detaillierten Untersuchung unterzogen. Die Anlage des vorliegenden Artikels kann keine tief greifende Gattungsdiskussion leisten. Kurz sei aber die terminologische Problematik im Falle der Kantate Caldaras geklärt: Zwar bezeichnet der Corriere ordinario das in Frage stehende Werk als „Spirituale Oratorio“ (CORRIERE, op. cit) und die Gazzetta di Bologna gar als „Pastorale“ (I-Bca Gazzetta Bolognese 1714 (9.1.1714), doch diesen Bezeichnungen stehen jene der Partitur und des Librettos gegenüber, die die Komposition eindeutig als „Cantata“ ausweisen (Vgl. GB-Lam MS 46, 1r sowie GINI und CALDARA, op. cit.). Stellt man das Werk in den Kontext der römischen Kompositionen Caldaras, so verdeutlich sich, dass Caldara (vgl. A-Wgm 400 (4)) wie seine Kopisten (vgl. D-MÜs SANT Hs 796 sowie D-MÜs SANT Hs 759) als auch die erhaltenen Abrechnungen der Casa Ruspoli (vgl. zu den Abrechnungen stellvertretend für andere KIRKENDALE, 2007, op. cit., S. 467-468, Eintrag 181) bei der Bezeichnung der Werke exakt zwischen Oratorium und Kantate unterscheiden. Vergleicht man zudem die erhaltenen Weihnachtskantaten Caldaras mit den in Rom entstandenen Oratorien, so zeigt sich, dass letztere zwei- bzw. dreiteilig gestaltet sind, die Kantaten hingegen keine solche Gliederung vorsehen. Die Länge der jeweiligen Kantate entspricht mit neun bis 13 Arien ca. der Hälfte eines Oratoriums, das im Regelfall um die 24 Arien aufweist. Schließlich sei zumindest erwähnt, dass auf Basis der Kantatentexte und der Rollenkonzeption im Bereich des dramaturgischen Aufbaus Unterschiede zu den Oratorien festgestellt werden können. Vgl. zu Aufbau und Inhalt der Kantaten die Ausführungen unten. ADAMI, op. cit., S. 101.

21 Vgl. ibid. Zur Gestaltung dieser Tischaufbauten (Trionfi) wurden auch Beschreibungen gedruckt, die die theologische Auslegung der Skulpturen beinhalteten. Vgl. GIANTURCO, op. cit., S. 6-7 sowie MARX, 1992, op. cit., S. 164-166. Für 1713 konnte keine Beschreibung aufgefunden werden.

Der Hinweis auf „,virtuose Sinfonie“ kann hier zweierlei bedeuten: Entweder wurde damit 
der kaiserliche Botschafter Ercole Turinetti und der Contestabile Colonna die Kardinäle zum Abendessen, welches im angrenzenden Appartement Albanis mit „Tavole ornate di Trionfi istorici“ ${ }^{23}$ vorbereitet war. Der knappen Beschreibung des Corriere ordinario ist zu entnehmen, dass die Musikdarbietung nicht allein den Kardinälen vorbehalten war. Die Anwesenheit von Diplomaten und Mitgliedern des römischen Adels kann auf Basis dieser Quelle belegt werden. Mit Hilfe des Librettodrucks der Weihnachtskantaten für den Papsthof wurde aller Wahrscheinlichkeit nach ein noch weiterer Adressatenkreis erreicht. Vor allem für jene Texte, die nicht allein das Weihnachtsfest und das übliche Papstlob ${ }^{24}$ thematisierten, sondern auch mit der damaligen politischen Situation in Verbindung zu bringen sind, wäre dies von besonderer Relevanz. Zur Verbreitung dieser Librettodrucke liegt bis dato keine Untersuchung vor, so dass zur Rezeption außerhalb des Papsthofes keine Aussagen getroffen werden können.

\subsection{Weihnachtskantaten für Fürst Francesco Maria Ruspoli}

Musikalische Aufführungen im Palazzo Ruspoli fanden üblicherweise im Rahmen der sonntäglichen Conversazione statt, für die Caldara während seiner Amtszeit als Maestro di cappella zwischen 1709 und 1716 an die 180 Kantaten verfasste. Während dieser Veranstaltungen fanden Vertreter des römischen Hochadels und des Klerus, aber auch Gesandte und Reisende zusammen, hörten Musik, spielten Karten und widmeten sich, angeregt durch die Lektüre von Avvisi, der Konversation. ${ }^{25}$ Neben den regelmäßig stattfindenden kleineren, musikalisch umrahmten Conversazioni verlangte der Karneval, der neben der Fastenzeit mit dem abschließenden Osterfest und Ferragosto am 15. August einen Höhepunkt des römischen Gesellschaftslebens bildete, eine dem Anlass angemessene musikalische

auf die Instrumentalbegleitung der Kantate verwiesen oder es wurden zusätzlich zur Kantate als „Sinfonie“ bezeichnete Instrumentalwerke gespielt. Sehr oft stammten diese dann von einem anderen Komponisten als jenem, der die Vokalkomposition lieferte. Eine vergleichbare Aufführungspraxis findet sich bei den Weihnachtsfeierlichkeiten der Arkadier, die unter der Protektion von Kardinal Pietro Ottoboni standen. Für die Feier des Weihnachtsfestes 1716 ist beispielsweise ein Ablauf in den Atti Arcadici überliefert, der vorsah, dass am Beginn eine „,nobilissima Sinfonia opera del defunto Arcomelo“, d. h. Arcangelo Corelli, gespielt wurde. Darauf folgten eine Oration sowie eine Ekloge, an die sich der erste Teil einer „,vaghissima cantata a due voci di soprano e contralto", komponiert von Gaetano Boni auf Basis eines Textes von Antonio Ottoboni, anschloss. Nach weiteren Rezitationen mehrerer Arkadier folgte der zweite Teil der Kantate. - Die Cantata à due voci liegt in der Henry Watson Music Library (Manchester): GB-Mp Ms. Q532Br53. Vgl. zum Ablauf und zum Verfasser des Kantatentextes die Angaben in: I-Ra, Atti Arcadia III (1712-1721) Ragunanza 231, Per la solennità della Festa del SS. ${ }^{\text {mo }}$ Natale. Al X. dopo il X. di Posideone Andante l'Anno IV. dell'Olimpiade DCXXIII.

23 A-Wn Il Corriere Ordinario 1714 (17.1.1714).

24 Vgl. zum Papstlob MARX, 1992, op. cit, S. 178-179.

25 Vgl. ZEDLER, Andrea. Antonio Caldaras Kantatenschaffen zwischen römischen Conversazioni und dem Zeremoniell des Wiener Hofes. In Studien zur Musikwissenschaft, 2013, 57, S.117-140, hier: S. 125-132. KIRKENDALE, 2007, op. cit., S. 56-106. 
Ausgestaltung. Weihnachten gehörte zweifellos zu den wichtigsten Ereignissen im römischen Jahreslauf, wurde jedoch in der Casa Ruspoli nur für relativ kurze Zeit mit speziell für diesen Anlass komponierten Werken begangen. Anders als für die Oratorien der Fastenzeit und die Commedie während des Karnevals sind für die Weihnachtskantaten nur äußerst spärliche Informationen überliefert; für manche Jahre weist, abgesehen von der Kopie des Notenmaterials, nichts auf eine Aufführung hin. ${ }^{26}$

Kirkendale hat in ihrer grundlegenden Studie zu den römischen Oratorien Caldaras auch Kantaten einzelner Weihnachtsfeierlichkeiten zugeordnet und verzeichnet für die Jahre von 1712 bis 1715 sieben Werke. ${ }^{27}$ Bei der erneuten Sichtung der erhaltenen Abrechnungen und Quittungen der Casa Ruspoli zeigten sich aber Schwierigkeiten bei der Zuordnung des Notenmaterials zu konkreten Aufführungsdaten. Die Abrechnungen, auf denen Kirkendales Annahmen basieren, beinhalten lediglich Hinweise auf Notenkopien oder bezahlte Musiker. Sie als Beleg für eine tatsächlich erfolgte musikalische Weihnachtsfeier zu verstehen bzw., wie es Kirkendale beispielsweise für das Jahr $1713^{28}$ vornimmt, von der Anzahl der auf den Abrechnungen angeführten Sängern auf konkrete Vokalbesetzungen zu schließen, wirft - wie gezeigt werden soll - Fragen auf. Im Folgenden wird ein Vorschlag für die chronologische Einordnung der Kantaten nach Aufführungsanlässen vorgelegt, wobei es in einigen Fällen notwendig war, inhaltliche Aspekte der Kantatentexte zu analysieren, um eine chronologische Einordnung zu ermöglichen.

Die chronologisch erste erhaltene Weihnachtskantate Caldaras erscheint in diesem Zusammenhang noch am unproblematischsten, da sie einem Aufführungsdatum mit hoher Wahrscheinlichkeit zugeordnet werden kann. Es handelt sich hierbei um Caldaras Neuvertonung des bereits $1703^{29}$ für den Palazzo Apostolico entstandenen Librettos Vaticini di pace ${ }^{30}$ von Paolo Gini für Weihnachten 1712. Francesco Lanciani hatte am 21. Dezember für die Cantata a 4 [...] per il SS. ${ }^{m o}$ Natale die Stimmen von „Pace, Amor divino, Giustizia, Core umano,

$26 \mathrm{Zu}$ den Oratorien und Commedie, die im Hause Ruspoli aufgeführt wurden, finden sich z. T. detaillierte Abrechnungen, die eine Rekonstruktion der Aufführungs-, aber auch der Probendaten ermöglichen. Vgl. KIRKENDALE, 2007, op. cit., S. 56-106, 446-478.

Die Rekonstruktion basiert auf den publizierten Abrechnungen von Caldaras Werken für die Casa Ruspoli in: ibid.

28 Vgl. ibid., S. 89.

291703 war das Libretto durch den aus Lucca stammenden Komponisten Domenico Bottari vertont worden, dessen Partitur bisher aber nicht wiederaufgefunden werden konnte. Der Kantatentext wurde für die Neuvertonung von 1712 komplett übernommen, lediglich die Schlussarie von 1703 entfällt. Somit endet die Kantate Caldaras mit der Arie Amor Divinos Quanto dolce, quanto cara.

30 Vgl. GINI, Paolo. Vaticini di Pace. Componimento per Musica. Da cantarsi nel Palazzo Apostolico. Rom: Luca Antonio Chracas, 1703 [cit. 2014-01-02]. URL: < http://www. mdz-nbn-resolving.de/urn/resolver.pl?urn=urn:nbn:de:bvb:12-bsb10578629-1 >. 
conc[erti]no, VV Co[ncert]o gr[oss] “" kopiert ${ }^{31}$, so dass eine Aufführung nur im Anschluss an dieses Datum in Frage kommt. Naheliegend ist der 25. Dezember, der in diesem Jahr auf einen Sonntag fiel - demjenigen Wochentag, an dem gewöhnlich Ruspolis Conversazioni stattfanden. Hierin mag der Grund für die erste Aufführung einer speziellen Weihnachtskantate liegen, denn zwischen 1705 und 1717, Ruspolis aktivster Zeit als Musikmäzen, fiel der Weihnachtstag nur ein einziges Mal, nämlich im Jahr 1712, auf einen Sonntag. Den 25. Dezember als Aufführungstag bestätigt zudem die heute in der Santini-Sammlung der Diözesanbibliothek Münster überlieferte Partitur der Kantate, die ebenfalls von Francesco Lanciani erstellt wurde. Sie ist mit dem Hinweis ,detto giorno Natalitio“ im Titel versehen: „Vaticini di pace, Cantata a quattro Voci con Violini, Per il S[antis]simo Natale, del Sig[no]r Antonio Caldara, Composta per S[ua] $\mathrm{E}$ [ccellenza] il Sig[nor]r P[ri]n[ci]pe Ruspoli, nell' anno 1712 per il d[et]to giorno Natalitio".32 Über die Abrechnung der bei Ruspolis Conversazione üblichen Kartenspiele kann zusätzlich der Nachweis erbracht werden, dass eine solche Zusammenkunft tatsächlich stattgefunden hatte: Domencio Castrucci, der für die Spielkarten im Hause Ruspoli zuständig war, verzeichnet für den 25. Dezember zwei Sets für Kardinal Joseph-Emmanuel de la Trémouille, der regelmäßig die Conversazioni bei Ruspoli besuchte. ${ }^{33}$

Im Gegensatz zur Kantatenaufführung im Palazzo Apostolico finden sich über den Ablauf der weihnachtlichen Conversazione bei Ruspoli in den Quellen nur wenige Hinweise. ${ }^{34}$ Vergleicht man die Zusammenkünfte im Palazzo Ruspoli mit jenen Weihnachtsfeiern bei Kardinal Pietro Ottoboni, kann zumindest davon ausgegangen werden, dass das übliche Kartenspiel nach der Musik angesetzt war. ${ }^{35}$ Auch die Zusammensetzung des Sängerensembles für diese Kantate wirft Fragen auf, die aufgrund der Haushaltsdokumente nicht geklärt werden können, denn Abrechnungen für Sänger und Musiker sind nicht erhalten. Mit dem Hausensemble, das Ruspoli unterhielt, konnte die für vier Solisten komponierte Kantate aber nicht angemessen aufgeführt werden, denn in diesem Zeitraum waren lediglich zwei Sängerinnen fest angestellt. Es waren dies die Sopranistin Anna Maria di Piedz, genannt Mariuccia, die entweder die Rolle der Pace oder des Amor divino gesungen haben dürfte und Agnesa Corsie, die als Altistin vermutlich als

31 I-Rasv, Fondo Ruspoli Marescotti. Filza delle Giustificazioni del Libro Mastro. Tomo II, B 55, 1713 (fasc. 13).

32 Die Partitur liegt heute in der Santini-Sammlung der Diözesanbibliothek Münster. CALDARA, Antonio. Vaticini di pace. D-MÜs SANT Hs 796.

33 Vgl. I-Rasv, Fondo Ruspoli Marescotti. Filza delle Giustificazioni del Libro Mastro. Tomo II, B 54, 1712 (fasc. 136).

34 Die konsultierten Avvisi und Gazette erwähnen dieseAufführungen bei Ruspoli nicht.

35 Vgl. beispielsweise den Bericht des bayerischen Gesandten Alessandro Clemente Scarlatti zu der Aufführung der Weihnachtskantate bei Kardinal Ottoboni, D-Mhsa, Kasten schwarz 7494 (11.1.1716). 
Giustitia besetzt worden war. ${ }^{36}$ Offen muss die Frage bleiben, wer die übrigen Gesangspartien übernommen haben könnte.

Für die Ruspolischen Weihnachtsfeierlichkeiten der folgenden Jahre ist die Datierungsfrage einer möglichen Aufführung wesentlich diffiziler als im Falle der Vaticini di pace. In chronologischer Folge müsste nun die von Caldara am 6. Januar 1713 datierte autographe Partitur der Weihnachtskantate Amarilli vezzosa besprochen werden. Sie ist mit einer Sopran- und zwei Altstimmen besetzt. Da die Stimmen und die Partitur jedoch erst im Dezember 1714 kopiert wurden, wird auf deren (möglichen) Aufführungsanlass weiter unten eingegangen.

Daher gilt es im Folgenden, mit der bereits im Abschnitt zu Caldaras Kantatenkomposition für den Papsthof erwähnten Zahlung an vier Sänger und je einen Violinisten und einen Violaspieler fortzufahren. ${ }^{37}$ Nicht nur die niedrigen Gagen der Sänger machen die These Kirkendales, wonach es sich um die Besetzung einer „Christmas cantata $a 4^{\star 638}$ handeln soll, unwahrscheinlich, auch die übrigen erhaltenen Dokumente sprechen eher gegen eine Kantatenaufführung für vier Solisten zu Weihnachten 1713 - zumindest im Rahmen einer Conversazione: Spielkarten wurden lediglich für Sonntag, den 10. Dezember 1713, abgerechnet, und auch die Bottiglieria, die für die Getränke bei der Conversazione zuständig war, machte zwar für den Weihnachtstag Ausgaben geltend, die dort verzeichneten „sei boccali“" wurden jedoch - so der Beleg - während der Probe zu Caldaras Oper Tito e Berenice konsumiert, die für den Karneval bestimmt war. Sie hatte am 8. Januar 1714 im Teatro Capranica ihre Premiere. ${ }^{39}$ Da zudem der 24. Dezember, der Tag an dem Caldaras Vo' piangendo e sospirando im Palazzo Apostolico gegeben wurde, auf einen Sonntag fiel, ist davon auszugehen, dass an diesem Tag keine Conversazione stattfand. Des Weiteren hatten zu dieser Zeit die Vorbereitungen für den Umzug der Familie Ruspoli vom Palazzo Bonelli in den neuen Palazzo am Corso bereits begonnen, die das Musikleben des Folgejahres erheblich beeinträchtigen sollten. ${ }^{40}$ Auf Basis der genannten Indizien erscheint die Aufführung einer - wie von Kirkendale entsprechend zugeschriebenen - so groß besetzten Kantate zu Weihnachten 1713 nicht plausibel.

Im darauffolgenden Jahr, 1714, wurde der Kopist Lanciani am 31. Dezember für zwei mit Weihnachten in Verbindung stehenden Arbeiten bezahlt. Es waren dies Amarilli vezzosa und die Cantata a 2 per il SS. Natale mit den Rollen Delia

36 Instrumentalisten waren bei Ruspoli in unterschiedlicher Weise engagiert: Neben den als $\mathrm{Ca}$ merieri fest angestellten Musikern Domenico und Pietro Castrucci, wurde das Ensemble mit jährlich oder vierteljährlich bezahlten Musikern ergänzt. Nur zu besonderen Anlässen mussten Musiker für einzelne Aufführungen extra bezahlt werden. Vgl. KIRKENDALE, 2007, op.cit., S. 437-444.

Vgl. Anm. 18.

Vgl. KIRKENDALE, 2007, op. cit, S. 89,

Vgl. I-Rasv, Fondo Ruspoli Marescotti. Filza delle Giustificazioni del Libro Mastro. Tomo II, B 56, 1713 (fasc. 155).

Vgl. KIRKENDALE, 2007, op. cit., S. 89. 
und Dori. ${ }^{41}$ Auf Basis der Abrechnungsbücher konnte eine Conversazione auf den 30. Dezember 1714 datiert werden, ${ }^{42}$ bei der eventuell eine oder beide Kantaten auf dem Programm gestanden haben könnten. Da weitere Indizien fehlen, kann über eine Aufführung aber allenfalls spekuliert werden. Bemerkenswert ist jedoch, dass die bereits im Januar 1713 von Caldara vorgelegte Kantate Amarilli vezzosa einer Revision unterzogen wurde..$^{43}$ Der Vergleich beider Textfassungen zeigt, dass bei der ersten klar das pastorale Sujet und damit die Schäfer im Zentrum stehen, die nach Bekanntwerden der Geburt Jesu als erste zur Krippe eilen und ihre einfachen Geschenke übergeben. Die thematische Verbindung des Schäfersujets mit der Weihnachtsgeschichte könnte ein Hinweis auf eine Aufführung im Kreise der Accademia dell'Arcadia sein, inszenierten sich die Mitglieder des literarischen Zirkels, deren Hauptförderer Ruspoli war, doch als Hirten. Zudem hatten sich die Arkadier das Christuskind zum Protektor gewählt, dem sie sich besonders verbunden fühlten. ${ }^{44}$ Naheliegend wäre demnach eine Aufführung im Rahmen der arkadischen Weihnachtsfeier in der Cancelleria Apostolica gewesen, die erstmals 1711 von Kardinal Pietro Ottoboni, dem Arkadierkollegen Ruspolis, ausgerichtet und ebenfalls mit Kantaten begangen worden war. ${ }^{45} \mathrm{Zwar}$ passt der Inhalt von Caldaras Kantate gut zum Argomento, der während der Versammlung bei Ottoboni vorgetragen wurde (Thema: „Perché il nascimento di Cristo Signor nostro si manifestasse prima d'ogni altro a' Pastori“"46), die Feier hatte jedoch bereits am 4. Januar 1713, also zwei Tage vor Fertigstellung des Autographs stattgefunden. ${ }^{47}$ Es ist daher möglich, dass die Kantate erst 1714, während der

41 Vgl. I-Rasv, Fondo Ruspoli Marescotti. Filza delle Giustificazioni del Libro Mastro. Tomo II, B 58, 1714 (fasc. 83). Das Notenmaterial zur Cantata a 2 konnte bisher nicht ausfindig gemacht werden.

42 Vgl. I-Rasv, Fondo Ruspoli Marescotti. Filza delle Giustificazioni del Libro Mastro. Tomo I, B 59, 1715 (fasc. 11).

43 Vgl. das autographe Material in A-Wgm 400 (4) sowie die Kopie in D-MÜs SANT Hs 759. Hier sei kurz auf die interessanten Abänderungen hingewiesen: Die Rolle Dametas ist trotz Beibehaltung der Altlage komplett umgearbeitet worden und insgesamt wurde die Kantate um je drei Rezitative und Arien erweitert.

44 Vgl. CRESCIMBENI, Giovanni Mario. Storia dell'Accademia degli Arcadi istituita in Roma l'anno 1690. Rom 1712, Reprint, Bulmer: London, 1805.

45 Vgl. I-Ra Atti Arcadia II (1696-1712) Ragunanza Particolare Al IV. d. Poseidone stante.

46 LEONIO, Vincenzo. Perché il nascimento di Cristo Signor nostro si manifestasse prima d'ogni altro a' Pastori. Ragionamento del medesimo Uranio Tegeo fatto a' 4 di Gennaio 1713 nella Cancelleria Apostolica, Capanna dell'Acclamato Crateo Ericinio [Pietro Ottoboni], ove gli Arcadi sogliono ragunarsi ogni anno a celebrare la sollenità del Santiss. Natale, lor Tutelare. In Prose degli Arcadi, Vol. I, hrsg. von Giovanni Mario Crescimbeni. Rom: Antonio de Rossi, 1718, S. 363-383.

47 Vgl. Atti Arcadici III (1712-1721) Al III dopo il X. di Poseidone Andante l'anno IV. dell'Olimpiade DCXXII. Im Folgejahr fand bei Ottoboni keine Weihnachtsveranstaltung statt. Vgl. ibid. Collegio al IV di Posideone stante l'anno 1 dell'Olimpiade DCXXIII ab A. 1. Olimp. VI Anno IV. Eine Aufführung bei Ruspoli beispielsweise zu Epiphanias 1713 konnte auf Basis der Quellen nicht nachgewiesen werden. 
datierbaren Conversazione vom 30. Dezember, in der umgearbeiteten Variante erklang. In dieser zweiten Fassung wurde das Libretto durch das Hinzufügen von drei Arien und den dazugehörigen Rezitativen vor dem Schlussduett um fast ein Drittel erweitert. Während Anspielungen auf den Papst in der autographen Fassung eine untergeordnete Rolle spielen, sind diese in der erweiterten Variante das wichtige Moment. Dadurch konnten nicht nur der Auftraggeber Ruspoli, sondern auch die Mitglieder der arkadischen Akademie, verkörpert durch die auftretenden Schäfer, dem Papst abermals ihre Treue und Ergebenheit zu Beginn eines neu anbrechenden Krieges, nämlich des - später so bezeichneten - venezianischösterreichischen Türkenkrieges (1714-1718) demonstrieren.

Weitgehend im Dunkeln bleiben die Feierlichkeiten für Caldaras letztes Weihnachtsfest bei Ruspoli im Jahr 1715, auf die erneut nur kopiertes Stimmenmaterial von Lanciani hinweist. Die Cantata a 5 per il SS. Natale fordert „3 angeli, pastore, Herode" - eine Rollenkonstellation, die bisher noch in keiner der überlieferten Partituren ausgemacht werden konnte. ${ }^{48}$

Den letzten Eintrag zu Weihnachtskantaten von Caldara für Ruspoli geben die Haushaltsbücher am 26. Februar 1716 mit der Rechnung von Francesco Lanciani über eine Partitur der Cantata a 4 per il SS.mo Natale an. ${ }^{49}$ Es handelt sich hierbei mit hoher Wahrscheinlichkeit um die zu Dokumentations- bzw. Sammlungszwecken erstellte Kopie von Vaticini di pace. ${ }^{50}$ Einen direkten Hinweis darauf gibt das genannte Datum „nell anno 1712“ auf dem Titelblatt der in der SantiniSammlung überlieferten Partitur. ${ }^{51}$

Überblickt man Ruspolis gesamte aktive Zeit als Mäzen von circa 1700 bis zu seinem Tod 1731, so ist unverkennbar, dass es sich bei den im Palazzo Ruspoli aufgeführten Weihnachtskantaten um ein auf Caldaras Amtszeit beschränktes Phänomen handelt. So hatten die für Ruspoli vor Caldara tätigen Komponisten Bernardo Gaffis ${ }^{52}$ und Georg Friedrich Händel keine entsprechenden Werke komponiert. Zwar wurde Händels Kantate Arresta il passo (HWV 83) verschiedentlich mit Feierlichkeiten bei Ruspoli am 25. Dezember 1706 und der, damals im Sommer stattfindenden, arkadischen Weihnachtsfeier in Verbindung gebracht, doch vermag die Umdeutung des explizit arkadisch-pastoralen zu einem christlichen Sujet im

48 I-Rasv, Fondo Ruspoli Marescotti. Filza delle Giustificazioni del Libro Mastro. Tomo II, B 60, 1715 (fasc. 118).

49 I-Rasv, Fondo Ruspoli Marescotti. Filza delle Giustificazioni del Libro Mastro. Tomo I, B 61, 1716 (fasc. 17).

50 CALDARA, Antonio. Vaticini di pace. D-MÜs SANT Hs 796. Zum Jahreswechsel 1715/1716 werden zahlreiche Werke Caldaras erneut kopiert, was mit seinem Weggang aus Rom nach Wien im Frühjahr 1716 zusammenzuhängen scheint.

51 Die Nennung eines konkreten Aufführungsjahrs im Titel einer Kantate ist außergewöhnlich, könnte aber durch die relativ große zeitliche Distanz zwischen erfolgter Aufführung und der Herstellung der Kopie begründet sein.

52 Bernardo Gaffi widmete Ruspoli 1700 einen der für Rom seltenen gedruckten Kantatenbände. Ein Exemplar ist heute in der Santini-Sammlung der Diözesanbibliothek überliefert. Vgl. D-MÜs SANT Dr 125. 
Falle dieser Kantate wenig zu überzeugen, und auch die Datierung der Kantate ist in der Forschung umstritten. ${ }^{53}$ Aus dem Zusammenhang gerissen, kann der „dio bambin“ zwar durchaus als Christuskind interpretiert werden, die Anrede „cieco dio" und der pastorale Kontext verweisen aber eindeutig auf den antiken Liebesgott Amor. Berthold Over zieht den Ansatz ebenfalls in Zweifel, ,da es in der Kantate keine weiteren Anspielungen auf die christliche Weihnachtsgeschichte gibt und im Gegenteil gegen Schluss Fillide Aminta (und nicht dem göttlichen Kind) ihre Liebe versichert (,La mia gioia, il mio bene altri non è che Aminta') “".54

Nach Caldaras Amtsende wurde seinem Nachfolger Francesco Gasparini 1716 die Ehre zuteil, die Cantata da recitarsi nel palazzo Apostolico la Notte del SS. ${ }^{\text {mo }}$ Natale auf ein Libretto von Antonio Baldini zu verfassen. ${ }^{55}$ Für Weihnachtsfeiern in Ruspolis Kreis der Conversazione lassen sich indes keine entsprechenden Nachweise mehr erbringen. Weder trägt eine der Kompositionen Gasparinis einen diesbezüglichen Titel, noch geben die überlieferten Notenmaterialien oder Dokumente darauf hindeutende Anhaltspunkte. ${ }^{56}$ Damit scheinen die musikalisch gestalteten Weihnachtsfeiern bei Ruspoli zu einem Abschluss gekommen zu sein, entließ er doch im Sommer 1718 den Großteil seiner Musiker und widmete sich fortan hauptsächlich dem Bau der Chiesa parrocchiale e collegiata di Santa Maria della Presentazione in Vignanello. ${ }^{57}$

53 Vgl. KIRKENDALE, Ursula. The Ruspoli documents on Handel. In Journal of the American Musicological Society, 1967, 20, S. 222-273. Gegen Kirkendales Datierung argumentiert FABRIS, Dinko. The ,Aria a 2 Sirene' (Rinaldo HWV 7a): A Neapolitan Emblem. In Händel-Jahrbuch 2011, LVII, S. 223-247. Warren Kirkendale verteidigt die Hypothese seiner Frau in: KIRKENDALE, Warren. Zu Handschriften von Händel und Caldara in der SantiniSammlung. In Studi musicali, Nuova serie, 2013, IV/1, S. 61-76, hier S. 63-66.

54 OVER, Berthold. Die Texte von Händels italienischen Kammerkantaten. In Händels Kirchenmusik und vokale Kammermusik (Das Händel-Handbuch 4), hrsg. von Michele Calella und Hans-Joachim Marx. Laaber: Laaber Verlag, 2012, S. 344-360, hier S. 358-359. Vgl. jüngst dazu auch RIEPE, Juliane. Händel vor dem Fernrohr. Die Italienreise. Beeskow: ortus (Studien der Stiftung Händel-Haus 1), 2013, S. 169.

55 Vgl. GASPARINI, Francesco und BALDINI, Antonio. Cantata da recitarsi nel palazzo Apostolico la Notte del SS.mo Natale nel Palazzo Apostolico l'anno MDCCXVI. Rom: Stamperia della Reverenda Camera Apostolica, 1716. [cit. 2014-01-02]. URL: <http://books.google. com/books?vid=IBNR:CR000610965>.

56 Lisa Navach vermutet zwar, dass Weihnachten während Gasparinis Amtszeit ebenfalls im Rahmen der Conversazione gefeiert werde, kann aber keine konkreten Stücke nachweisen. Vgl. NAVACH, Lisa. „Cantando il duol dell'alme“. Analisi ed edizione critica delle cantate con violini di Francesco Gasparini, 2 Bd., Diss. masch, Università di Pavia, 2004, S. 53.

57 Ruspoli entlässt seine Hausmusiker im Juni 1718. Vgl. NAVACH, op. cit, S. 31. Nach diesem Zeitpunkt sind in Bezug auf die Musik vor allem Abrechnungen zu Theaterlogen nachweisbar, und ein Großteil der Investitionen bezieht sich auf den Kirchenbau in Vignanello. Dieser Bau steht im direkten Zusammenhang mit der von Ruspoli und seinem Onkel Kardinal Galeazzo Marescotti maßgeblich (auch monetär) geförderten Beatifizierung Giacinta Marescottis. Vgl. SAMERSKI, Stefan: „Wie im Himmel, so auf Erden“? Selig- und Heiligsprechung in der Katholischen Kirche 1740 bis 1870. Stuttgart: Kohlhammer, 2002, S. 224-231. 
Zusammenfassend lässt sich feststellen, dass die Weihnachtskantaten im Hause Ruspoli nie den Glanz und die Außenwirkung seiner Oratorienaufführungen erreichten. Ein Grund dafür mag in den Vorbereitungen für die Commedie des Karnevals liegen, die Ruspoli bereits während der Weihnachtszeit aufnahm. Aus welchen Beweggründen der Fürst in den Jahren zwischen 1712 und 1715 dennoch Weihnachtskantaten aufführen ließ und welche Botschaft hinter den allegorischen und pastoralen Texten stand, wird in den folgenden Abschnitten erörtert.

\section{Die Textgrundlage der Weihnachtskantaten und deren inhaltliche Verknüpfungen}

Wie bereits in der Einleitung angesprochen, ist Crescimbenis Hinweis auf die „correnti cose d'Italia“ für die Interpretation der Texte von erheblicher Bedeutung. Demzufolge sind auch Paolo Ginis Texte mit Blick auf die politischen Geschehnisse im Zuge des Spanischen Erbfolgekrieges und der besonderen Situation, in der sich Rom nach dem Erdbeben von 1703 befunden hatte, zu interpretieren. ${ }^{58}$ Am Beispiel der Kantate Vaticini di Pace wird anhand einer Textanalyse exemplarisch gezeigt, wie Gini die zentrale Botschaft der Geburt Christi auf die konkrete historisch-politische Situation bezog.

Nicht allein der Titel, auch die Wahl der allegorischen Figuren - Core Umano, Core Divino, Pace und Giustitia - geben einen ersten Hinweis auf die in der Kantate zu diskutierende Friedensthematik: Die verzweifelte Suche des menschlichen Herzens nach dem ersehnten Frieden ist Ausgangspunkt der dramatischen Entwicklung des Textes. Pace lässt nicht lange mit der Erklärung auf sich warten, dass der sündige Mensch selbst Verursacher seiner beklagenswerten Situation sei und verknüpft dies mit der Forderung nach Sündenbereinigung und Hinwendung zu Gott. Durch Amor Divinos Bitte um Gnade, die er Pace gegenüber mit dem Hinweis auf das göttliche Geschenk der Geburt des heiligen Kindes begründet, könnte die Kantate gleich zu einem guten, friedvollen Ende finden, doch es tritt die erbarmungslose Giustitia hinzu. Sie nimmt die zentrale Rolle der Konflikttreiberin ein, indem sie vehement für die Bestrafung der Menschheit plädiert. Core Umano ist nun zwischen Verzweiflung und Hoffnung hin- und hergerissen. Das Geschenk der göttlichen Liebe aber hat nachhaltig Eindruck auf Pace gemacht. Sie zeigt ein Einsehen mit den Menschen und bemüht sich, auch Giustitia zum Einlenken zu bewegen. Doch erst unter dem Eindruck der friedvollen Strahlkraft des Jesuskindes lässt Giustitia von ihrem harten Urteil ab und schließt mit Pace einen Bund, der das menschliche Herz rettet und der Welt Frieden bringt.

58 Vgl. zu den Auswirkungen des Krieges und des schweren Erdbebens von 1703 auf das Musikleben Roms jüngst FRANCHI, op. cit., 2012, S. 96-98. Franchi listet im Anschluss an seinen Artikel jene Werke aus dem römischen Umfeld auf, die mit dem Spanischen Erbfolgekrieg in Verbindung zu bringen sind. Interessanterweise findet sich dort die Vo' piangendo e sospirando-Kantate Ginis, aber nicht Vaticini di Pace, die - wie die folgende Textanalyse zeigen wird - der Liste hinzugefügt werden kann. Vgl. ibid. S. 112-116. 
Die Textanalyse zeigt, dass Anspielungen auf den Spanischen Erbfolgekrieg im gesamten Kantatentext zu finden sind: Gleich zu Beginn konfrontiert Gini seine Zuhörer mit der Frage nach dem Frieden: „Bella pace, ove sei?“59 Der zusätzliche Verweis auf den Kriegsgott Mars und die beim Krieg eingesetzten Musikinstrumente, Pauken und Trompeten, die dem menschlichen Herz den Schlaf rauben, veranschaulichen die verzweifelte Lage, in der sich die „care spiagge latine", also die Apenninenhalbinsel, befand. ${ }^{60}$ Als Überleitung zur Arie Bella pace riedi a noi setzt Gini an den Schluss des ersten Rezitativs eine Repetitio, in der die zentrale Frage nach dem Frieden erneuert wird. ${ }^{61}$

Giustitias Text ist der vom Librettisten intendierten Rollenausrichtung gemäß voll von martialischem Vokabular: Mit dem in ihrem ersten Rezitativ Che dici, che pretendi Amor Divino ausformulierten Aufruf an die Waffen zur Rache des wahren Gottes (Su dunque alle straggi / Impiagate, uccidete, armi, saette / Fate del giusto dio giuste vendette) positioniert sich Giustitia als Gegenspielerin des sündigen Menschen. Noch schärfer fällt die Attacke in der nachfolgenden Arie Da nemica ultrice spada aus, gipfelt diese doch in dem Wunsch der Tötung des ungerechten Menschen durch das feindliche Racheschwert (Da nemica ultrice spada / Pera sì, l'iniquo cada / Del furor vittima esangue). Auch nach dem Dialog Gravi tanto l'offese mit Core Umano, der in der verzweifelten Lage Barmherzigkeit erbittet, bleibt Giustitia hart und legt in ihrer Arie Sì, sì perirà dar, dass die entsetzliche Qual beispielgebend für die Welt sei (Sì, sì perirà / E l'orrido scempio / Di specchio, d'esempio / Al mondo sarà). Die Kantate befindet sich hier am dramaturgischen Höhepunkt.

Ginis Lösung des Konflikts basiert auf zweierlei: Im ersten Schritt verbreitet Core Divino mit den Worten „Non paventar, habbi di me fidanza / Trionferò di Lei, di sua possanza“ sowie in der Folge mit „Non perirai, no, no; sarà tuo scudo

59 Auch in den Rime degli Arcadi finden sich Werke, die auf den Spanischen Erbfolgekrieg rekurrieren. Gini selbst war zwar kein offizielles Mitglied der Arkadier, dennoch lassen sich seine italienischsprachigen Texte auf Basis ihrer Thematik, Struktur und Versgestaltung dem arkadischen Umfeld zuordnen. Bemerkenswert hierbei ist die thematische und zumindest für den Beginn strukturelle Ähnlichkeit zu Casaregis Rima „E quando sia, che bella Pace amica“, die ebenso eine einleitende Frage nach dem Frieden und einen Verweis auf Mars bringt: „E Quando fia, che bella Pace amica / con aureo piede a noi sen torni, e il freno / Marte morendo, il fier suo corso arreste?; CASAREGI, Giovanni Bartolommeo (Eristo Faresio). E quando sia, che bella Pace amica. In Rime Degli Arcadi. Tomo Quinto. All'Illustriss. ed. Eccellentiss. Signore La Signora D. Maria Isabella Cesi Ruspoli Principessa di Cerveteri, \&., hrsg. von Giovanni Mario Crescimbeni. Rom: Antonio de Rossi, 1717, S. 262-266. Vgl. hierzu auch: NECCHI, Rosa. Marte nel Bosco Parrasio. La Rappresentazione della guerra nelle Rime degli Arcadi. In Per violate forme. Rappresentazioni e linguaggi della violenza nella letteratura italiana, hrsg. von Fabrizio Bondi und Nicola Catelli. Lucca: Maria Pacini Fazzi Editore, S. 47-60.

60 Im Zuge des Spanischen Erbfolgekrieges begannen im Frühjahr 1701 die Kampfhandlungen auf italienischem Gebiet. Vgl. hierzu: PRESS, Volker. Kriege und Krisen. Deutschland 1600-1715. München: C.H. Beck, 1991, S. 457-465.

61 Der vollständige Kantatentext wie auch der anderen hier besprochenen Kantaten findet sich auf der Internetplattform CLORI: www.cantataitaliana.it. 
/ Il petto d'un fanciullo ancor ch'ignudo" Hoffnung. Die Passagen verweisen eindeutig auf den Ausruf des Engels „Fürchtet euch nicht!“ und die Ankündigung der Geburt Christi im Lukasevangelium (2, 10-11). Im zweiten Schritt setzt Gini (die bereits besänftigte) Pace ein, um Giustitia an das Jesuskind (,quel pargoletto") zu erinnern. Giustitias Worte in der Arie Già mi sento intenerir sind Ginis Interpretation des Kantaten-Mottos „Et in terra pax“, das sich am Titelblatt des 1703 gedruckten Librettos befindet. Mit „Chi bambin sul fieno giace / Non più guerra, ma vuol pace / Risanar, e non ferir / Vuol Amor, non crudeltà“ formuliert er Giustitias Einsicht aus, dass Frieden statt Krieg, Heilung statt Verletzung und Liebe statt Grausamkeit vorherrschen sollen.

Eine Zuspitzung auf die Friedensaussage erfährt Caldaras Werk überdies mit der einzigen Veränderung des Librettos von 1703: Die Schlussarie von Pace Quanto vago, quanto amato entfällt und mit ihr Paces erneut formulierte Freude über das göttliche Kind. Die Version von 1712 endet hingegen mit Amor Divinos Arie Quanto dolce, quanto cara. Diese Arie ist der Freude über den Frieden gewidmet und lässt das Stück mit den Worten Pace gradita - dem willkommenen Frieden - enden. Geschickt verwebt Gini die von der Geburt Christi ausgehende Friedensbotschaft mit dem Lob auf Papst Clemens XI., indem er Amor Divino die Worte clemente und clemenza in den Mund legt, um Pace für sich zu gewinnen. Clemenza allein solle regieren, denn nur durch sie könne der Erde Friede geschenkt werden ( $\mathrm{Su}$ dunque la Clemenza / Regni solo e trionfi al reo condona / Et al mondo fedel, Pace ridona). Noch eindrücklicher erfolgt die Stilisierung des Papstes als Friedensbringer durch die zweimalige Referenz auf das Papstwappen des Kantatentextdruckes (siehe Abb.1):

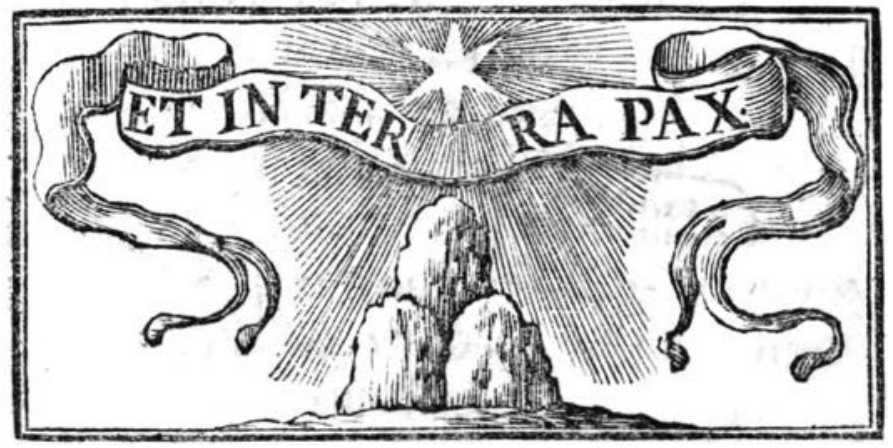

Abb. 1: Vignette mit Wappenemblem Papst Clemens XI. aus dem Libretto Vaticini di pace

Amor Divino weist gegen Ende der Kantate darauf hin, dass über den drei Bergen schon der Stern bewundert werde könne, der von der regierenden Barmherzigkeit blühe. Pace setzt gleich darauf mit der Hoffnung ein, dass sich die Vaticini di Pace, die Prophezeiungen des Friedens, für den Vatikan bewahrheiten werden, denn - und hier kommt es zur erneuten Andeutung auf das Papstwappen - der 
barmherzige freundliche Stern, der gleichzeitig an den Stern von Bethlehem erinnert, zeige den Weg zum Frieden (La clemente amica stella / Qual accesa in ciel facella / Via di pace additerà). Mit diesen Referenzen auf das Wappen verband Gini in geradezu plakativer Weise den himmlischen Friedensbringer mit seinem weltlichen Stellvertreter, an dessen Autorität hierbei erinnert werden sollte.

Auch die äußere Form des Librettodrucks unterstützt die Aussage des Textes, indem die zentralen Worte - wie Clemente, Pace und Giustitia - durch Kapitälchen hervorgehoben und in der abschließenden Abbildung prägnant verbildlicht werden (siehe Abb. 2).

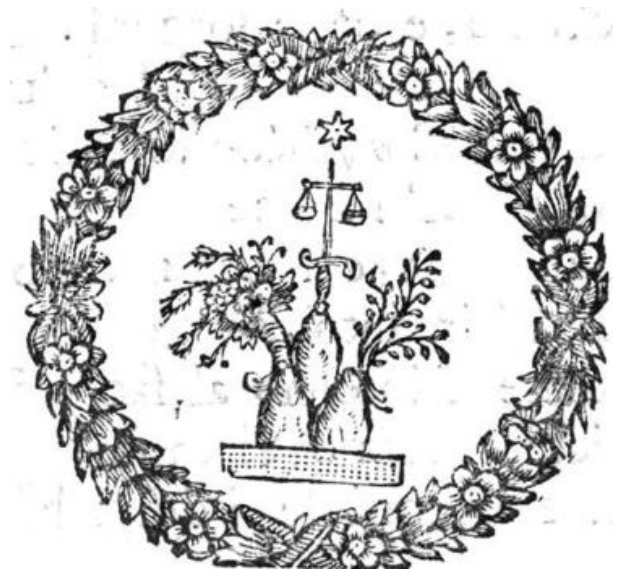

Abb. 2: Vignette mit Wappenemblem Papst Clemens XI. aus dem Libretto Vaticini di pace

Ginis 1713 geschriebener Text der Cantata da recitarsi la notte del SS. ${ }^{m o}$ Natale, Vo'piangendo e sospirando, für die Weihnachtsfeierlichkeiten am Papsthof ist auf vielerlei Ebenen mit der 1703 entstandenen Vaticini di pace verknüpft. Diese Knotenpunkte, aber auch inhaltliche Unterschiede der beiden Kantaten Ginis, gilt es im Folgenden zu erläutern.

Auf der Ebene der Besetzung kam es zu einer Reduktion auf drei allegorische Figuren, wobei erneut Amor Divino und Giustitia die Handlung tragen. Keine Schwierigkeiten bereitet die Gleichsetzung der neu eingeführten Natura Umana mit der ehemals eingesetzten Personifikation des Core Umano, wird doch auch Natura Umana am Beginn der Kantate im Zustand der Verzweiflung gezeigt. Zudem bleibt Giustitias Rollenkonzeption unverändert: An Amor Divino, der als Konfliktlöser auftritt, richtet Giustitia die Forderung nach einem Menschen als Gott. Dieser Forderung kommt Amor Divino nach, der Retter der Welt wird geboren, Giustitia weicht zurück. Amor Divino verlangt für sein Geschenk Ehrfurcht und Treue von Natura Umana und entlässt sie mit der Botschaft von Erlösung und Frieden. 
Gemeinsam ist beiden Kantaten die inhaltliche Fokussierung auf die Friedensbotschaft, die von der Menschwerdung Christi ausgeht. Im Fall der Kantate Vo' piangendo e sospirando zeigt die Textanalyse, dass Gini die Situation wesentlich subtiler zeichnet. Auch die Referenzen auf den Papst und das Kriegsgeschehen sind quantitativ zurückhaltender eingesetzt: Natura Umana ist sich ihrer Schuld schon von Beginn an bewusst und benennt den Grund ihrer Verzweiflung: „Errai ohimè peccai / contro Dio, contro me stessa / Piango la mia caduta / L'innocenza perduta". Die sie durch die Kantate begleitenden Affekte sind wie bei Vaticini di pace zuerst Verzweiflung und Reue, ${ }^{62}$ dann Hoffnung und Freude. Zuversichtlich gestimmt wird Natura Umana vor allem im dialogisch angelegten Rezitativ Voci soavi, e care, das Gini mit einer - die zentrale Aussage des Textes unterstreichenden - Repetitio ausstattet. Der Textautor betont hierbei die Hoffnung auf Frieden nach langem Kummer:

Natura Umana:

Doppo sì lunghi affanni

Spero per te goder la Pace amata.

Amor Divino:

Non più, non più t'affanna,

Spera per me goder la Pace amata.

Giustitia, deren Duktus erneut dem Kriegsvokabular (u. a.: Stragi, e sangue grida ogn'ora / Al mio Dio l'antico error) entstammt, stellt sich dieser Hoffnung entgegen und führt die Kantate zum dramaturgischen Höhepunkt, indem sie Tribut fordert: „Pagar altri non può, che un uomo dio“. Amor Divino willigt ein, erfüllt den Wunsch nach der Fleischwerdung Gottes (Farò, sì, sì, che scenda / Dal sommo cielo, e prenda / Spoglia mortal fatt'uom l'istesso Dio) und verweist gleichzeitig auf die Erlösung der Menschheit durch Christi Tod (E con la morte sua morto il delitto). Hierin liegt der zentrale Unterschied zu Vaticini di pace, bei der der Hinweis auf die Geburt Christi ausreicht, um Pace sowie Giustitia zu beruhigen. Nach der Lösung des Konflikts sind es die Worte des Friedens, der Herrlichkeit und der Freiheit, die als das finalisierende Motto der Kantate eingesetzt werden. Im abschließenden Chor werden diese mit dem Papstlob verbunden, und der Pontifex wird erneut als potenter Friedensstifter dargestellt. Wieder spiegelt sich das Papstwappen in den Worten stella, tre monti und Clemente. Hinzugefügt ist noch der Verweis auf den Familiennamen des Papstes, Albani, durch ,in Ciel Alba ridente".

62 Mit der ersten Textzeile seiner Kantate „Vo’ piangendo e sospirando“ wählt Gini einen Ausganspunkt, der unweigerlich eine Assoziation zu Petrarcas Sonett „I' vo piangendo i miei passati tempi“ (Canzoniere 365) hervorruft. Petrarcas Rima wird den Reuegedichten untergeordnet und ist der Redesituation nach eine Ansprache an Gott. Dabei sei, so Küpper, das ,anzitierte Schema des itinerarium mentis - Vorleben in Sünde, Einsicht und Umkehr" erfüllt. KÜPPER, Joachim. Petrarca. Das Schweigen der Veritas und die Worte des Dichters. Berlin, New York, N.Y.: de Gruyter \& Co, 2002, S. 162. 
Pace, Gloria e Libertà.

Per noi splende amica Stella;

Spunta in Ciel Alba ridente,

Luce a noi reca più bella

Da tre Monti un sol Clemente.

Auch der Text des anonym gebliebenen Autors der Amarilli Vezzosa-Vertonungen lässt die zentrale Friedensbotschaft von Weihnachtskantaten nicht vermissen. Das typisch pastorale Sujet, das die in den beiden Fassungen der Amarilli vezzo$s a$ dargestellte Szene der Entdeckung und Verehrung des Jesuskindes durch die arkadischen Hirten Dafni, Amarilli und Dameta aufgreift, kann als „,christliche Interpretation der arkadischen Welt ${ }^{\star 63}$ gesehen werden. Zentral für die Friedensbotschaft sind die Gaben einzelner Schäfer an das Jesuskind. Ihm werden u. a. zwei Tauben, die wiederum einen Olivenzweig überbringen, geschenkt. Dieses Zeichen des Friedens zaubert - so die Arie Dametas - ein süßes Lächeln auf das kindliche Gesicht (Della pace al caro segno / Dolce riso nel bel viso / Mosse il vago pargoletto). Das Lächeln wird als Hinweis gelesen, dass Jesus zum Frieden auf Erden beitragen werde: „Dir parea dall'alto regno / Scendo anch'io dall'amor mio / A dar pace all'alme eletto“. Die Freude der Hirten über die friedensstiftende Geburt Christi wird in der Pastoralarie Dafnis mit der übergroßen Freude darüber verbunden, dass das göttliche Kind unter den einfachen Hirten geboren wurde (Se un nume che ha di stelle in trono adorno / È nato fra le rustiche capanne).

Wie bereits im Abschnitt zu den Weihnachtskantaten Caldaras für Principe Ruspoli angedeutet, erfährt der Kantatentext durch die Erweiterung in der zweiten Fassung von 1714 eine neue inhaltliche Komponente. Hier ist vor allem die Papstverehrung in Rechnung zu stellen, die mit dem Verweis auf das Papstwappen sinnfällig gemacht wird: So kann sich beispielsweise Dafni gut vorstellen, dass der glückliche Hügel Ursprung des allgemeinen Wohls sei (Scorger ben si potea / Che in te nascer dovea / Felice colle, la comun salute) und lobt diesen als den schönsten von allen (Del cielo sei più bella, Vezzosa collinetta). Die erste neu angelegte Arie (Il sol co'i primi rai / Sempre t'illuminò, / Bella collina; / E nubiloso mai / Per te non ritornò / Dalla marina), deren Text nichts mit der zuvor gezeichneten pastoralen Szene gemein hat, könnte auch den Anhaltspunkt für die Adaption des Textes geben: Am 8. Dezember 1714 hatten die Türken Venedig den Krieg erklärt und Clemens XI., der von den Absichten der Pforte erfahren haben dürfte, schickte bereits zuvor „Gesandte an den Kaiserhof und an die Höfe der deutschen Fürsten, um sie gegen den Erbfeind der Christenheit zum Kampfe aufzurufen" ${ }^{\prime 64}$. Zeitgleich hatte die Neuorganisation der päpstlichen Flotte an der Adria begonnen, um Zeichen eines türkischen Angriffes frühzeitig zu erkennen. ${ }^{65}$

\footnotetext{
63 MARX, 1992, op. cit., S. 178.

64 THEUER, Franz. Brennendes Land, Kuruzzenkriege. Ein historischer Bericht, Ein BöhlauSonderband. Wien: H. Böhlau, 1984, S. 327.

65 Vgl. Archivio della R. Società Romana di Storia Patria. Vol. XXIII, Fasc. I-II. 1900. S. 239-244.
} 
Die in der Arie ausgedrückte Hoffnung, dass sich der Himmel nie vom Meer her verfinstern möge, könnte sich daher auf den aufkeimenden Krieg gegen die Türken beziehen und somit als Referenz auf das zeitgenössische politische Geschehen gelesen werden.

Für die Textgrundlage der Kantaten kann zusammenfassend festgehalten werden, dass die „correnti cose d'Italia“ im Besonderen in jenen von Paolo Gini verfassten Texten eine hervorstechende Rolle spielen. Gerade wenn Gini die Friedensbotschaft des Weihnachtsfestes mit den Elementen des Papstwappens wie Dreiberg und Stern verbindet, wird das in Rom propagierte Bild des Papstes als Friedensstifter und -vermittler in den Jahren der Friedensverhandlungen zum Spanischen Erbfolgekrieg deutlich. Marx' Einschätzung in Bezug auf $\mathrm{Va}$ ticini di Pace, dass ,Anspielungen auf politische Themen [...] wenn überhaupt - am Schluss des Textes in Verbindung mit der Huldigung des Papstes zu finden sind "66 , kann auf Basis der Ergebnisse der Textanalyse revidiert werden. Es zeigte sich, dass sich solche Referenzen durch den gesamten Kantatentext ziehen. Ähnlich verhält es sich bei der Kantate Vo' piagendo e sospirando, die - so konnte es der Textvergleich zeigen - inhaltlich an Vaticini di Pace orientiert ist und somit die Grundaussage des ursprünglich 1703 für den Papsthof entstandenen und 1712 im Auftrag von Ruspoli wiederaufgegriffenen Vaticini di Pace-Textes noch 1713 beibehält. Im Gegenzug dazu sind die beiden Fassungen der Amarilli vezzosa stärker vom arkadisch-pastoralen Sujet geprägt. Der inhaltliche Fokus liegt auf der Friedensbotschaft, und die Anbindung an das zeitgenössische Geschehen wird lediglich in der zweiten Fassung greifbar, die sich vermutlich auf das Zeugnis der Papsttreue des Auftraggebers am Beginn des Türkenkrieges bezieht. Aus diesem Grund können keine ausgeprägten inhaltlichen Verbindungslinien der Amarilli vezzosa zu den Gini-Kantaten gezogen werden. Sehr wohl bestehen solche jedoch - wie im Folgenden gezeigt wird - auf der Ebene der musikalischen Umsetzung von Caldara.

\section{Musikalische Korrelationen zwischen den Kantaten}

Bei vergleichender Betrachtung der drei erhaltenen Werke wird offensichtlich, dass Caldaras Weihnachtskompositionen einer bestimmten Erwartungshaltung gerecht werden mussten. Die Kantaten sind jeweils einteilig angelegt und weisen ohne Ausnahme eine (auch jeweils so bezeichnete) Sinfonia auf. Daran schließt sich die übliche Abfolge von monologischem oder dialogischem Seccorezitativ und Arie an. ${ }^{67}$ Mit nur zwei Ausnahmen in Vaticini di Pace sind die Arien als Da-Capo Arien ausgeführt. Die Tonarten, die Caldara für die Sinfonie wählt entsprechen dem weihnachtlichen Sujet und sind in F-Dur bei Amarilli vezzosa und Vo piangendo

66 MARX, 1992, op. cit., S. 175.

67 Zur musikalischen Struktur, der Tonartenwahl, Besetzung und Satzbezeichnung vgl. die Angaben auf CLORI: www.cantataitaliana.it. 
e sospirando bzw. in B-Dur bei Vaticini di Pace gehalten. Am Ende der Kantaten kehrt Caldara in allen Fällen in die Ausgangstonart der Sinfonie zurück.

Zentrales - und somit auch erwartetes - Element der Auftraggeber und der Zuhörerschaft ist die Einbindung der Pastorale, die ohne Ausnahme in die Sinfonie integriert ist. Da auf die Pastoralsätze in den Sinfonien von Amarilli vezzosa und Vo 'piangendo e sospirando weiter unten eingegangen wird, seien hier lediglich die augenfälligsten Gemeinsamkeiten summarisch festgehalten: Die Pastorale ist jeweils als Schlusssatz der Sinfonia gesetzt und weist in allen drei Fällen die üblichen Charakteristika auf: 12/8-Takt, über längere Abschnitte Einsatz des Basses als Orgelpunkt auf der Tonika und Dominante, Führung der ersten und zweiten Violine im Terzabstand sowie Verwendung von Synkopen und Punktierungen. Alle drei Pastoralsätze sind zweiteilig, wobei die einzelnen Teile mit Wiederholungen versehen sind.

Üblich war es auch, Pastoralarien in solche Werke einzubinden. Caldaras Pastoralarien lassen die typischen Ausprägungen dieses Arientypus nicht vermissen: Es ist wiederum der 12/8-Takt gewählt, das Tempo ähnelt sich durch die Wahl von Largo bzw. Larghetto, der Bass wird in Teilen als Orgelpunkt geführt und die Violinstimmen sind über längere Passagen hinweg im Terzabstand geführt. Die Pastoralarien stechen nicht allein auf Basis ihrer speziellen Ausgestaltung innerhalb der jeweiligen Kantate hervor, sie wirken auch als Ruhepol in der Abfolge der von Allegro-Arien dominierten Weihnachtskantaten.

Zur Illustration der Text-Musik-Beziehung seien stellvertretend zwei Beispiele herausgegriffen: Es handelt sich hierbei um je eine Rezitativ- und Arienvertonung aus Vaticini di Pace, bei der gezeigt wird, wie der Komponist den Vorgaben des Autors folgt, bzw. wie er die zentralen Aussagen des Textes mit musikalischen Mitteln verstärkt.

Bei Paces Rezitativ Su questo eccelso monte versieht Caldara Verszäsuren jeweils mit Pausen, so dass das Versschema beim Hören nachvollziehbar ist. Im dritten und siebenten Vers, die als Endecasillabi gestaltet sind, fügt Caldara durch Pausensetzung jeweils eine Binnenzäsur nach der siebenten Silbe ein. Bei der dritten Verszeile berücksichtigt der Komponist das Komma durch Pausensetzung, während der Vers eine Verschleifung bei nome und et vorsieht.

Su questo eccelso monte

Che da bugiardi vati

Ebbe l'antico nome, et hora il prende

Dagl'adorati, e santi

Infalibili oracoli divini

Spera vederne o core

Avverati di pace i vaticini.
7 A

7 B

$11 \mathrm{C}$

7 D

$11 \mathrm{E}$

$7 \quad \mathrm{~F}$

$11 \mathrm{E}$

Ohrenfällig sind Terz- bzw. Quartsprung der Singstimme bei divini und vaticini, die den Reim - und damit die göttlichen Prophezeiungen des baldigen Friedens - betonen. Als Kadenz setzt Caldara die vorgezogene Kadenz, die durch eine Fermate im Schlusstakt einen Ruhepunkt bringt, bevor die Arie La clemen- 
te amica stella in G-Dur und Da-Capo-Form einsetzt. Caldara verwendet hier die - oft gewählte - Besetzungsvariante Violine 1 und 2 unisono, Sopranstimme und Continuo. Oktavsprünge und punktierte Achtel dominieren und betonen den tänzerischen Duktus des 3/8-Taktes. Die Arie wird mit einem Ritornell eingeleitet, das insgesamt viermal erklingt. Das Ritornell gliedert sich in $4+4+7$ Takte, wobei die jeweiligen Abschnitte motivisch kontrastieren. Der erste Viertakter ist zu Beginn von Dreiklangszerlegungen geprägt, auf die nach einem Oktavsprung $\left(g^{6}-g^{\text {" }}\right)$ eine schrittweise Aufwärtsbewegung mit punktierten Sechzehnteln folgt. Der zweite Viertakter ist mit punktierten Sechzehntelketten gestaltet und schließt mit einem Oktavsprung $\left(\mathrm{g}^{\text {" }}-\mathrm{g}^{6}\right)$. Die ersten drei Takte des Siebentakters weisen eintaktige Motivzellen auf, die jeweils einen ohrenfälligen Triller auf der ersten Taktzeit vorsehen. Der vierte Takt leitet über zur Sequenz, die zu einem authentischen Ganzschluss führt und das Ritornell auf der Tonika beendet. Die Gesangsstimme setzt nun im nächsten Takt ein und trägt beinahe vollständig den Melodieverlauf des Ritornells vor, lediglich der Siebentakter wird nun auf einen Viertakter verkürzt, und erst im Anschluss folgt ein von punktierten Sechzehntelketten ausgeformter Siebentakter, der mit einer Kadenz hin zur Dominate abgeschlossen wird. Somit ergibt sich eine Aufteilung des ersten Gesangsteils in $4+4+4+7$ Takte. Charakteristisch für die Ariengestaltung Caldaras ist die oftmalige Verwendung des Tacet im Basso Continuo. So pausiert der Bass in dem von D-Dur dominierten Gesangsteil 2 (Schema: $4+4+6+5$ ) nach einem ersten Viertakter auch bei Wiederholung der zweiten Verszeile Qual accesa in ciel facella bis zum erneuten Einsatz des Ritornells. Während der Pause des Continuos werden Singbzw. Instrumentalstimme überwiegend im Terzabstand geführt. Erst bei Via di pace additerà kommt es zum Ausbruch der Violinstimme aus der Parallelführung, indem Caldara eine aufsteigende Linie setzt, die vom kleinen $g$ ausgehend über zwei Oktaven bis zum Höhepunkt $\mathrm{d}^{3}$ bei pace geführt wird.

Die Symmetrie der Textvorlage wird in der musikalischen Umsetzung exakt übernommen, indem im A-Teil die ersten drei inhaltlich verkettenden Verszeilen und im B-Teil die zweiten drei Verszeilen verarbeitet sind. Der Zusammenhang der zwei Blöcke wird dabei durch den Reim additerà / sorgerà hergestellt:

La clemente amica stella

Qual accesa in ciel facella

Via di pace additerà:

Da tre monti un'alba d'oro

A scoprir il gran tesoro

Chiara, e bella sorgerà

$\begin{array}{ll}8 & \text { A } \\ 8 & \text { A } \\ 8- & \text { B } \\ 8 & \text { C } \\ 8 & \text { C } \\ 8- & \text { B }\end{array}$

Caldara vertont für den A-Teil die ersten drei Verszeilen zweimal, wobei er jeweils den Versschluss des dritten Verses durch Wiederholung betont. Der Text des in e-Moll gehaltenen B-Teils wird lediglich mit Wiederholung der letzten Verszeile in Musik gesetzt. Der Gesangsteil im B-Teil ist wie folgt gestaltet: $4+4$ $+6+3$ Takte. Die musikalischen Einschnitte sind durch Binnenkadenzierungen 
wiederum so gewählt, dass die Versstruktur erkennbar bleibt. Bemerkenswert ist das Dreiklangsmotiv am Beginn des B-Teils, das das Eingangsmotiv der Arie zitiert, gleichzeitig aber den Verlauf umkehrt, so dass bei monti der höchste Punkt erreicht ist. Insgesamt ist die Arie durch ihren leichtfüßigen tänzerischen Rhythmus sehr eingängig komponiert. Durch die Wiederholung des Ritornells wird die Erinnerung an die vorgetragene erste Verszeile La clemente amica stella erleichtert - und dies nicht ohne Grund, wird damit doch die zentrale Aussage der Kantate, dass der barmherzige Stern (Papst Clemens) den Weg des Friedens zeige, musikalisch betont.

Die Pastorale bildet nicht nur ein zentrales Charakteristikum der Weihnachtskantaten an sich, sie schafft im Falle von Amarilli vezzosa und Vo' piangendo e sospirando auch eine direkte musikalische Verknüpfung. Pastorale und Pastoralarie beider Kantaten sind mehr als thematisch verwandt, sie weisen Entlehnungen auf, die in ihrer Klarheit einmalig in Caldaras Kantatenschaffen für Ruspoli sind. ${ }^{68}$ Das 1713 vollendete Autograph der Amarilli enthält die Pastorale als dreistimmigen Schlusssatz der Sinfonia, die geprägt ist von einem auf- und absteigenden Motiv mit Quart- und Terzsprüngen, das in den beiden Violinstimmen imitierend über einem ruhenden Bass geführt wird (siehe Abb. 3). ${ }^{69}$ Dieses Motiv wird unverändert in Vo 'piangendo e sospirando übernommen, aber um eine Violenstimme erweitert

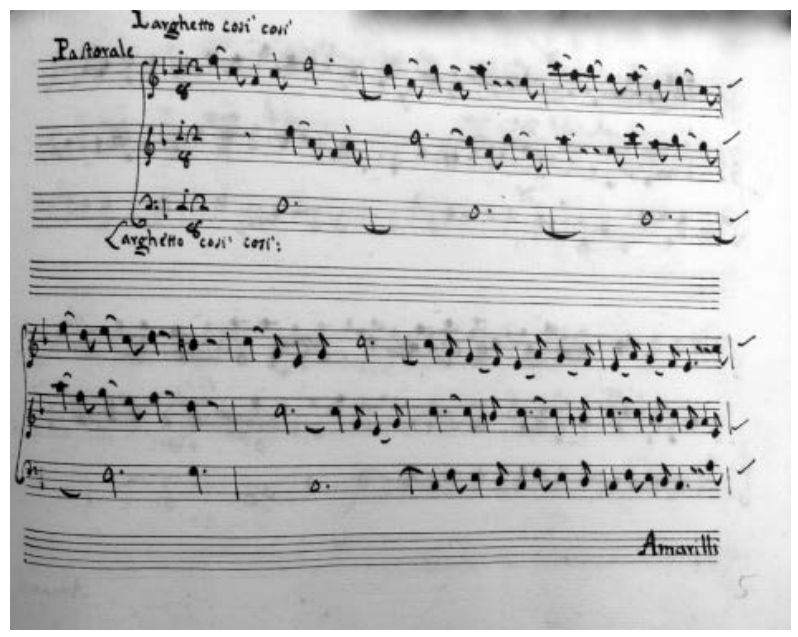

Abb. 3: Ausschnitt aus: Pastorale aus der Sinfonia der Kantate Amarilli vezzosa, D-MÜs SANT Hs 759 (1713/1714)

68 Im Rahmen des Dissertationsprojekts zu Caldaras Kantatenschaffen für Principe Ruspoli wurden die Werke mittels Incipitvergleich auf musikalische Zitate hin untersucht. Nach bisherigem Erkenntnisstand handelt es sich hier um den einzigen Fall direkten Zitierens eigener Werke. Ein Abgleich mit Caldaras Opernarien - vor allem derjenigen der Wiener Zeit - ist indes noch ein Desiderat der Forschung. Vgl. BOSCHUNG, Magdalena. Die Kantaten Antonio Caldaras für Principe Francesco Maria Ruspoli - Form und Funktion. Berlin: Merseburger, (M.A.R.S. - Musik und Aristokratie im Rom des Sei-/Settecento, 5) (in Vorbereitung).

69 Die erweiterte Abschrift der Amarilli vezzosa von 1714 übernimmt den dreistimmigen Satz der autographen Fassung unverändert. 
und als vierstimmiger Schlusssatz der Sinfonia verwendet (siehe Abb. 4). Zunächst verdoppelt die Viola nur die erste Violine, gewinnt dann aber zunehmend an Eigenständigkeit, ohne jedoch den Satz grundlegend zu verändern.

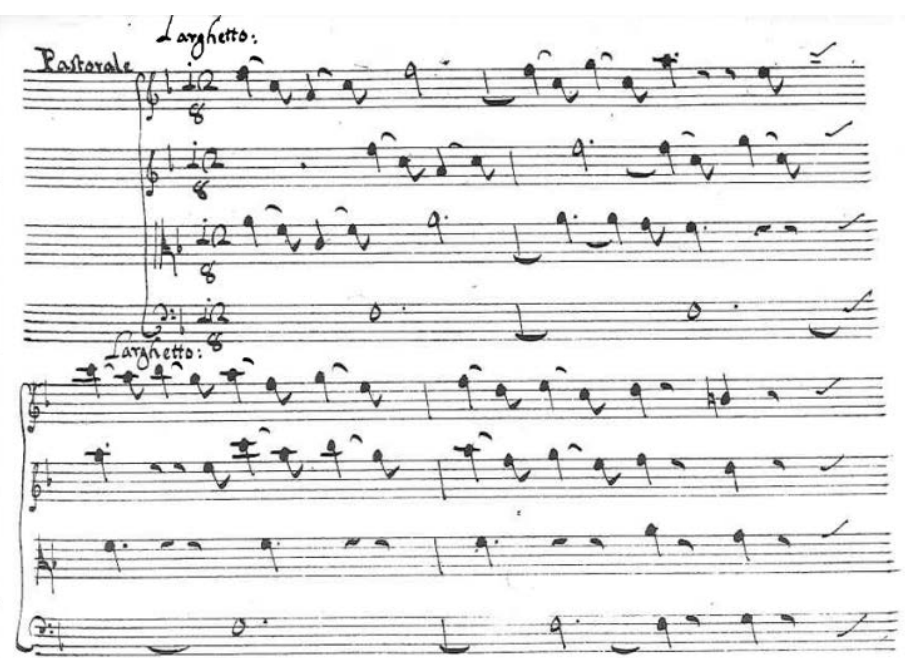

Abb. 4: Ausschnitt aus: Pastorale aus der Sinfonia der Kantate Vo' piangendo e sospirando, GB-Lam MS 46 (1713).

Die in den beiden Kantaten enthaltenen Pastoralarien Sempre risuoneranno (siehe Abb. 5) und Quel Pargoletto (siehe Abb. 6) sind in ihrer musikalischen Thematik ebenfalls identisch und unterscheiden sich in den Eingangstakten nur

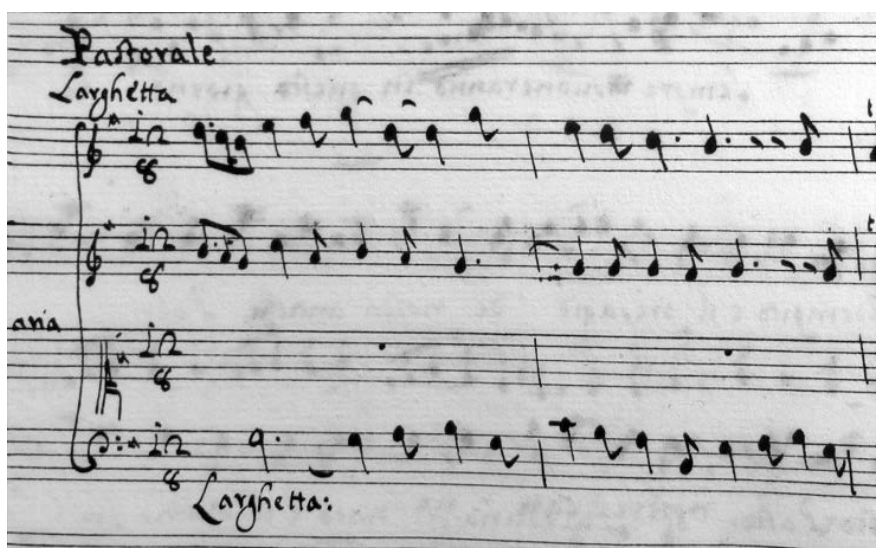

Abb. 5: Ausschnitt aus: Pastoralarie aus der Kantate Amarilli vezzosa, D-MÜs SANT Hs 759 (1713/1714) 
dadurch, dass Caldara in letztgenannter entsprechend der größeren Besetzung von Vo' piangendo e sospirando erneut eine Violenstimme ergänzt hat.

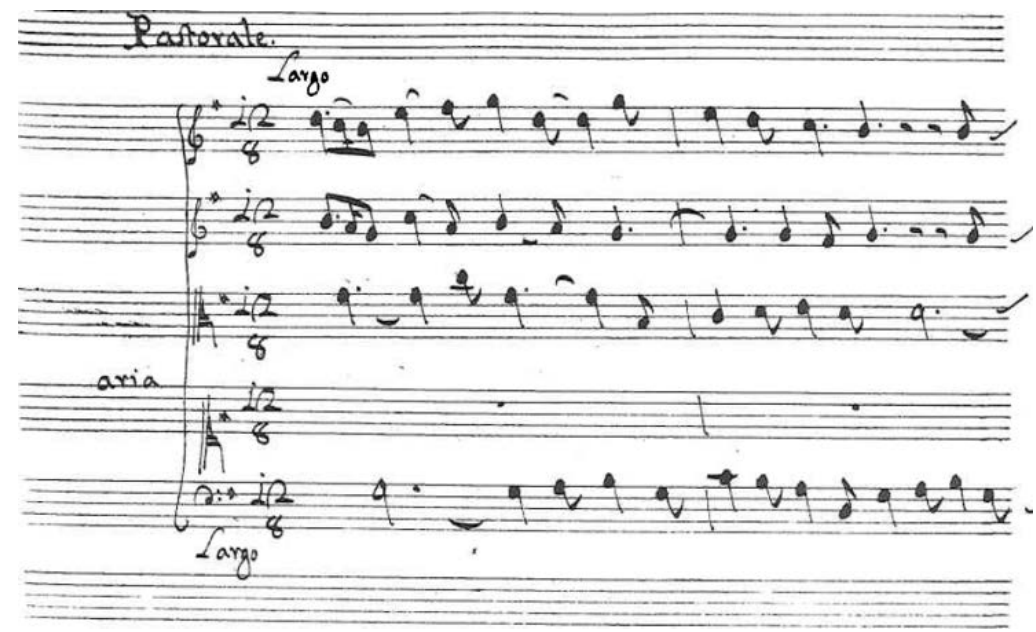

Abb. 6: Aussschnitt aus: Pastoralarie aus der Kantate Vo 'piangendo e sospirando, GB-Lam MS 46 (1713)

Mit dem Einsetzen der Singstimmen wurden aber größere Eingriffe in die musikalische Struktur nötig, da sich Caldara mit metrisch unterschiedlichen Textvorlagen konfrontiert sah. Während es sich in Sempre risuoneranno um regelmäßige Endecassillabi handelte, musste er das vorhandene motivische Material für die Arie in Vo 'piangendo e sospirando auf das Metrum der Quinari mit abschlieBendem Neunsilbler anpassen.

Sempre risuoneranno in questo giorno

$\begin{array}{ll}11 & \text { A } \\ 11 & \text { B } \\ 11 & \text { A } \\ 11 & \text { B }\end{array}$

Amarilli vezzosa $(1713 / 1714)$

Quel pargoletto

$5 \quad \mathrm{~A}$

Nudo, e negletto,

$5 \quad \mathrm{~A}$

Che nascer (nascer) volle in vil capanna

9 (11) B

Con voce amica

$5 \quad \mathrm{C}$

Palesi, e dica

$5 \quad \mathrm{C}$

Se il ver ti dice, ò amor t’inganna.

9 B

Vo 'piangendo e sospirando (1713) 
Der veränderten Schwerpunktsetzung begegnet Caldara mit auftaktigem Einsatz der Singstimme anstelle des volltaktigen Beginns in Sempre risuoneranno, der verkürzten Silbenzahl mit der Wiederholung des zweitaktigen Anfangsmotivs. Dank der Wiederholung des Wortes nascer erreicht er im letzten Vers des A-Teils ebenfalls elf Silben, was es ihm erlaubt, die Melodie der Vorlage zu übernehmen, zwar um einen halben Takt verschoben, aber in Tonhöhe und Rhythmus unverändert.

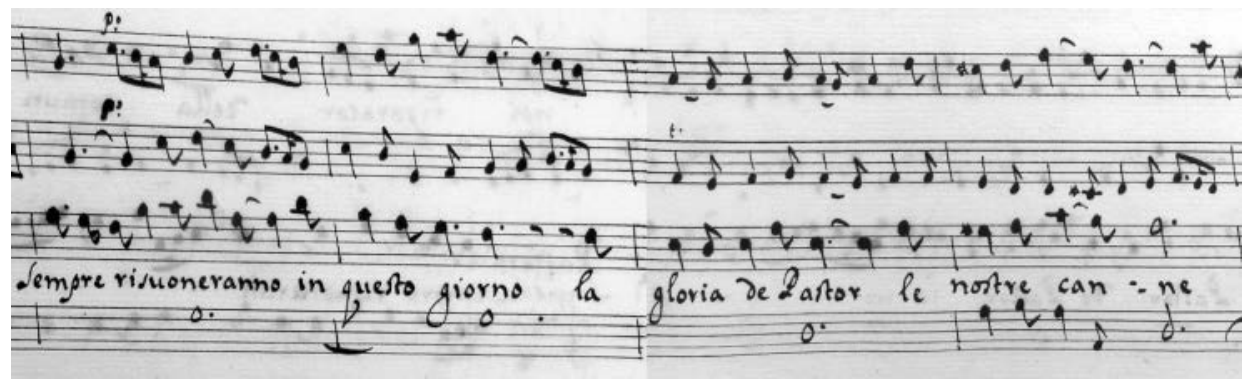

Abb. 7: Ausschnitt aus: Pastoralarie aus der Kantate Amarilli vezzosa, D-MÜs SANT Hs 759 $(1713 / 1714)$

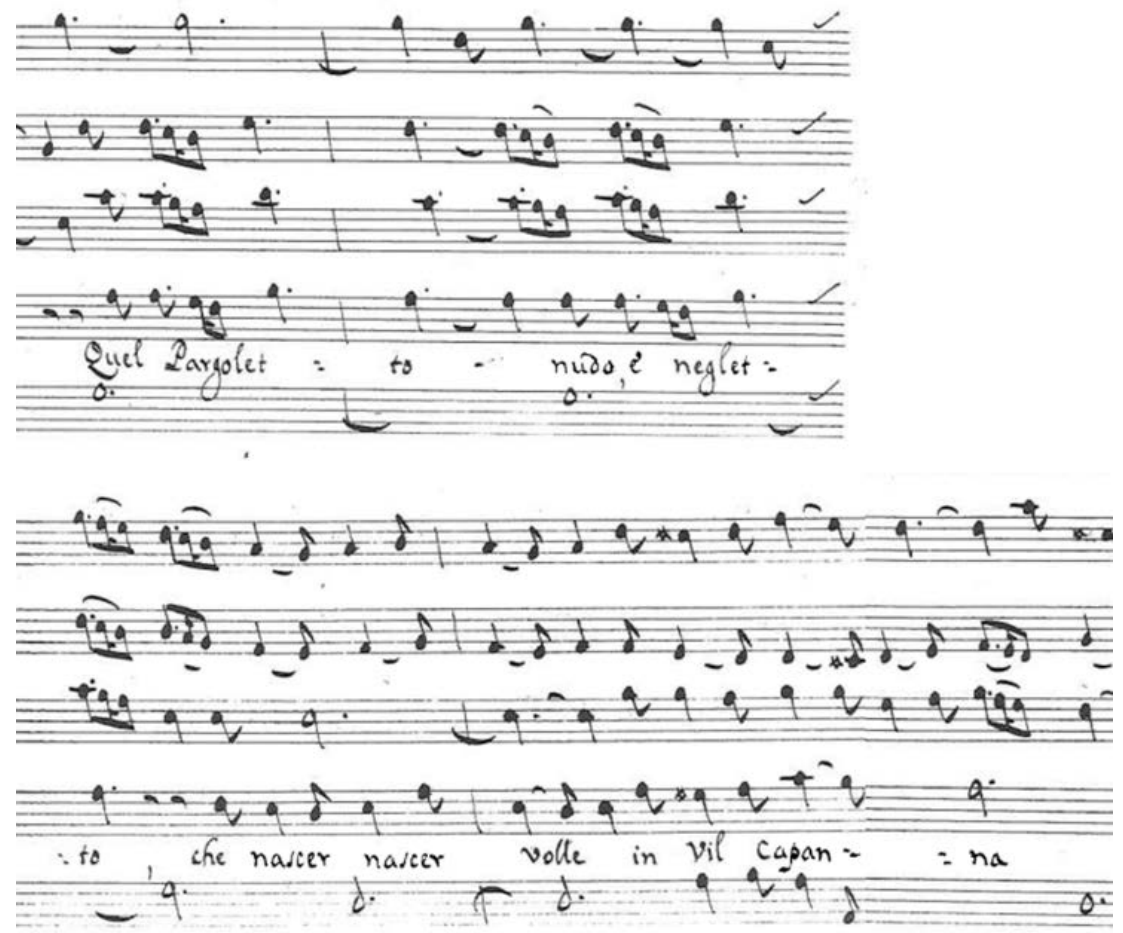

Abb. 8: Ausschnitt aus: Pastoralarie aus der Kantate Vo'piangendo e sospirando, GB-Lam MS 46 (1713) 
Mit den hier angeführten Ausschnitten sollte die musikalische Verwandtschaft der beiden Stellen belegt und an einem ersten Beispiel Caldaras Arbeitsweise bei Adaptionen innerhalb seines Kantatenrepertoires nachvollziehbar gemacht werden. Da das musikalische Zitat nach bisherigem Kenntnisstand in Caldaras Schaffen für Ruspoli einmalig ist und von einer zeitnahen Aufführung der Werke ausgegangen werden kann, scheint eine zufällige Übernahme unwahrscheinlich. Zwar kann über den Grund bis dato nur spekuliert werden, doch erscheint es plausibel, dass es sich bei der Kantatenkomposition für den Papsthof um eine bewusst gewählte Anspielung auf die erste Fassung der Amarilli vezzosa handelte, mit der der Komponist im Kontext der päpstlichen Aufführung auf seinen Dienstherrn Ruspoli verweisen wollte. Bei einer zweiten Aufführung der Amarilli vezzosa 1714 wäre die Melodie - nun geadelt durch die Verwendung in der Kantate für den Apostolischen Palast - zusammen mit dem neu eingefügten Papstlob ein musikalisches Element, um Ruspolis Treue und Ergebenheit gegenüber Papst Clemens XI. zu unterstreichen.

\section{Resümee}

Im Museo di Roma (Palazzo Braschi, Rom) ist gegenwärtig ein Ölbild ausgestellt, das eine Allegorie auf das Pontifikat Clemens XI. zeigt. Es stammt vermutlich von Giuseppe Chiari und ist zwischen 1712 und 1714 entstanden. ${ }^{70}$ Das Gemälde präsentiert den heiligen Petrus vor dem päpstlichen Thron mit AlbaniWappen, flankiert von der allegorischen Figur der Justitia, die mit der rechten Hand auf den Papststuhl weist. Petrus deutet hingegen auf die Figur des Friedens, die mit einem Ölzweig in der Hand im Begriff steht, vor der Figur des Glaubens herabzuschweben. In der linken unteren Bildecke sind römische Krieger dargestellt, die vor Pace und der Muttergottes mit dem Jesuskind, die rechts über dem päpstlichen Thron auf einer Wolke sitzen, zurückweichen. Das Bild konstruiert ganz offensichtlich eine ,celebrazione della pace riconquistata“71, und inszeniert dabei den Pontifex durch den Verweis auf das Familienwappen als Friedensstifter im Spanischen Erbfolgekrieg. Die - hier lediglich exemplarisch herangezogene - Bildkomposition ist nicht allein vom Thema her, sondern auch in ihren Details den Kantaten so eng verwandt, dass man die im selben Zeitraum entstandenen Werke Caldaras Vaticini di pace sowie Vo' piangendo e sospirando ins Bild gesetzt glaubt.

Schnettgers eingangs zitierte Einschätzung, wonach Rom während des Erbfolgekrieges zum Schlachtfeld der Propaganda geworden sei, gilt demnach nicht allein für die Kriegszeit im engeren Sinn, sondern gleichermaßen für die Jah-

\footnotetext{
70 LEONE, Rossella. Il Museo di Roma racconta la città. Roma: Gangemi, 2002, S. 72-73. Vgl. hierzu auch JOHNS, Christopher M. S. Papal Art and Cultural Politics: Rome in the Age of Clement XI. Cambridge: University of Cambridge Press, 1993, S. 7. Leider konnte bis dato der Auftraggeber dieses Werkes nicht eindeutig zugeordnet werden.
}

71 LEONE, op. cit., S. 72 
re der Friedensverhandlungen. In diesen Jahren entstanden in der Urbs aeterna Kunst- und Musikwerke, die die propazifistische Inszenierung des Papstes unterstrichen. In engem Zusammenhang mit dem intendierten päpstlichen Image steht - ausgehend von der Frage ob und in welcher Form Caldaras Weihnachtskantaten von den zeitgenössischen politischen Umständen beeinflusst wurden das Analyseergebnis jener Werke. Hierbei zeigte sich, dass Caldaras Neuvertonung des Librettos Vaticini di pace von Paolo Gini eine besondere Bedeutung zukommt. Zwar war es im Hause Ruspoli innerhalb der Conversazioni durchaus üblich, Kantaten eines Komponisten mehrfach aufführen zu lassen, doch stellt die Neuvertonung eines bereits 1703 gedruckten und von einem anderen Komponisten in Musik gesetzten Textes nach bisherigem Erkenntnisstand eine Ausnahme dar. Warum nicht ein neues Libretto verfasst wurde und warum die Wahl ausgerechnet auf diesen, 1712 bereits neun Jahre alten Text fiel, kann nur von dessen hohem Symbolgehalt abgeleitet werden. Vaticini di pace ist durchdrungen von Anspielungen auf den Spanischen Erbfolgekrieg und zeugt von der Bemühung, den Pontifex als Vermittler und Friedensbringer zu stilisieren - ein Bild, das zwar weder der politischen Realität von 1703 noch derjenigen von 1712 entsprach, wohl aber jenem Bild, das die Anhänger des Papstes in der Öffentlichkeit zeichnen wollten. Zieht man darüber hinaus Ruspolis gesellschaftliche und politische Situation im Jahr 1712 in Erwägung, so wird deutlich, weshalb sich gerade dieses Libretto zur Wiederaufnahme anbot: Ruspoli gehörte aus familienpolitischer Sicht zu den Nutznießern des Spanischen Erbfolgekrieges. Der bis dahin den Titel eines Marchese führende Ruspoli hatte 1708 auf eigene Kosten ein Regiment errichtet, das den Papst im Kampf gegen die kaiserlichen Truppen im Comacchio-Krieg (1708/09) unterstützte. Obwohl letztlich militärisch erfolglos, wurde Ruspoli zum Dank für seine Hilfeleistung und Treue gegenüber Clemens XI. 1709 in den Rang eines Principe erhoben. Nach dieser Phase des aktiven Eingreifens in das politische Geschehen - die infolge der propäpstlichen Haltung auch zu einer profranzösischen geführt hatte ${ }^{72}$ - und nach seiner Rangerhöhung war Ruspoli daran gelegen, seine politische Stellung erneut zu dokumentieren. In der Phase der Friedensverhandlungen, die nach dem Comacchio-Krieg den politischen Machtverlust des Papstes offenbart hatten, war eines der Mittel hierfür die Aufführung von Vaticini di pace. Sie brachte deutlich sowohl seine unveränderte Treue und Dankbarkeit als auch seine ideologische Verbundenheit mit dem Heiligen Vater zum Ausdruck. Darüber hinaus bot die Aufführung Ruspoli die Gelegenheit, sich in seiner neuen gesellschaftlichen Stellung als potenter Mäzen zu inszenieren, der seinen Maestro di cappella für seine private Weihnachtsveranstaltung ein Werk vom Rang und der Dimension der Kantaten am Papsthof komponieren lassen konnte. Ob es sich bei Vaticini di pace gewissermaßen um lena. Antonio Caldaras Serenata Il trionfo d'amore: Frankreichrezeption im Dienste adeliger Selbstdarstellung. In Die italienische Kantate im Kontext aristokratischer Musikpatronage, hrsg. von Berthold Over. Berlin: Merseburger (M.A.R.S. - Musik und Aristokratie im Rom des Sei-/Settecento, 3) (im Druck). 
eine Bewerbung für die Weihnachtskantate 1713, Vo 'piangendo e sospirando, für den Apostolischen Palast handelte, muss vorerst Spekulation bleiben. Es ist jedoch bemerkenswert, dass Caldara von Seiten des Papsthofes mit der Vertonung eines Librettos beauftragt wurde, das inhaltlich und dramaturgisch auffällig nahe an Vaticini di pace liegt. ${ }^{73}$

Solche inhaltlichen und dramaturgischen Verbindungslinien wurden im Laufe der Untersuchung des Textes freigelegt und die Anbindung an das zeitgenössische Geschehen nachgewiesen. Abschließend wurde bei der Musikanalyse gezeigt, dass zwischen der Kantate Vo' piangendo e sospirando und den beiden Fassungen der Amarilli vezzosa musikalische Korrelationen bestehen. Auf Basis der schmalen Quellenlage aus dem Umfeld der Kantatenkompositionen kann über die Gründe für die musikalischen Zitate lediglich spekuliert werden. Ein absichtsvoller Einsatz der Entlehnung scheint aber plausibel, schließlich konnte aus Sicht des Auftraggebers Ruspoli mit dem musikalischen Zitat in der zweiten Fassung der Amarilli vezzosa an die Aufführung der Kantate Vo' piangendo e sospirando im Palazzo Apostolico erinnert werden, was nicht zuletzt der Steigerung seines eigenen Ansehens gedient haben dürfte.

\section{Anhang 1: Antonio Caldaras römische Weihnachtskantaten}

\begin{tabular}{|c|c|c|c|c|}
\hline Kantatentitel & Datierung & Besetzung & Textautor & Quellen \\
\hline \multicolumn{5}{|l|}{ Vaticini di pace } \\
\hline & & $\begin{array}{l}\mathrm{S}(2), \mathrm{A}, \mathrm{T}, \mathrm{vl} 1, \\
\mathrm{vl} 2, \mathrm{bc}\end{array}$ & & \\
\hline & Textdruck:1703 & & Paolo Gini & D-Mbs L.eleg.m. 3800 \\
\hline & $\begin{array}{l}\text { Kantatenkopie } \\
\text { von Francesco } \\
\text { Lanciani: } \\
21.12 .1712\end{array}$ & & & $\begin{array}{l}\text { Die Kantatenkopie ist nicht } \\
\text { erhalten; Abrechung: I-Rasv, } \\
\text { Fondo Ruspoli Marescotti. Filza } \\
\text { delle Giustificazioni del Libro } \\
\text { Mastro. Tomo II, B 55, } 1713 \text { (fasc. } \\
\text { 13) }\end{array}$ \\
\hline & \begin{tabular}{|l|} 
mögliche \\
Aufführung: \\
25.12 .1712$, \\
Conversazione, \\
Palazzo Bonelli
\end{tabular} & & & $\begin{array}{l}\text { I-Rasv, Fondo Ruspoli Marescotti. } \\
\text { Filza delle Giustificazioni del Libro } \\
\text { Mastro. Tomo II, B 54, } 1712 \text { (fasc. } \\
\text { 136). }\end{array}$ \\
\hline
\end{tabular}

73 Vaticini di Pace ist nicht das einzige Zeugnis einer musikalisch-politischen Stellungnahme Ruspolis, die eng mit einem Treuebeweis an den Papst verbunden ist. Stellvertretend sei hier das Oratorium San Clemente papa e martire erwähnt, das Ruspoli erstmals 1704 und in den Jahren 1708, 1709 und 1712 wieder aufführen ließ. Das Werk rückte erstmals den heiligen Klemens ins Zentrum einer Oratorienhandlung. Bereits das Titelblatt des Librettos verknüpft die Wappenelemente des Albani-Papstes - Stern und Dreiberg - mit dem Inhalt des Oratoriums. Vgl. hierzu FRANCHI, 2002, op. cit., S. 283-284. 


\begin{tabular}{|c|c|c|c|c|}
\hline & $\begin{array}{l}\text { erneute } \\
\text { Kantatenkopie von } \\
\text { Francesco Lancani: } \\
\text { 26.2.1716 }\end{array}$ & & & $\begin{array}{l}\text { D-MÜs SANT Hs 796; I-Rasv, } \\
\text { Fondo Ruspoli Marescotti. Filza } \\
\text { delle Giustificazioni del Libro } \\
\text { Mastro. Tomo I, B 61, } 1716 \text { (fasc. } \\
\text { 17). }\end{array}$ \\
\hline \multicolumn{5}{|l|}{ Amarilli vezzosa } \\
\hline & & $\begin{array}{l}\mathrm{S}(1), \mathrm{A}(2), \mathrm{vl} 1, \\
\mathrm{vl} 2, \mathrm{bc}\end{array}$ & unbekannt & \\
\hline & \begin{tabular}{|l} 
Autograph (erste \\
Fassung): 6.1 .1713 \\
\end{tabular} & & & A-Wgm $400(4)$ \\
\hline & $\begin{array}{l}\text { Kantatenkopie von } \\
\text { Francesco Lanciani } \\
\text { (zweite Fassung): } \\
\text { 31.12.1714 }\end{array}$ & & & $\begin{array}{l}\text { D-MÜs/ SANT Hs 759, I-Rasv, } \\
\text { Fondo Ruspoli Marescotti. Filza } \\
\text { delle Giustificazioni del Libro } \\
\text { Mastro. Tomo II, B 58, } 1714 \text { (fasc. } \\
\text { 83). }\end{array}$ \\
\hline & $\begin{array}{l}\text { mögliche } \\
\text { Aufführung der } \\
\text { zweiten Fassung: } \\
\text { 30.12.1714, } \\
\text { Conversazione, } \\
\text { Palazzo Ruspoli } \\
\end{array}$ & & & $\begin{array}{l}\text { I-Rasv, Fondo Ruspoli Marescotti. } \\
\text { Filza delle Giustificazioni del Libro } \\
\text { Mastro. Tomo I, B 59, } 1715 \text { (fasc. } \\
\text { 11). }\end{array}$ \\
\hline \multicolumn{5}{|c|}{ Cantata da recitarsi la notte del SS. ${ }^{\text {mo }}$ Natale, Vo' piangendo e sospirando } \\
\hline & & $\begin{array}{l}\mathrm{S}(2), \mathrm{A}, \text { Chor } \\
(\mathrm{S}, \mathrm{A}, \mathrm{T}, \mathrm{B}), \mathrm{vl} 1, \mathrm{vl} \\
2, \mathrm{vla}, \mathrm{bc} \\
\end{array}$ & & \\
\hline & Textdruck: 1713 & & Paolo Gini & I-Rig Rar. Libr. Orat. 18. Jh. 164 \\
\hline & $\begin{array}{l}\text { Kantatenkopie } \\
\text { von Francesco } \\
\text { Lanciani, } \\
\text { spätestens } \\
\text { Dezember } 1713 \\
\end{array}$ & & & GB-Lam MS 46 \\
\hline & $\begin{array}{l}\text { Aufführung: } \\
\text { 24.12.1713, } \\
\text { Palazzo Apostolico }\end{array}$ & & & $\begin{array}{l}\text { GB-Lam MS 46, I-Bca Gazzetta } \\
\text { Bolognese } 1714 \text { (9.1.1714), } \\
\text { A-Wn II Corriere Ordinario } 1714 \\
(17.1 .1714)\end{array}$ \\
\hline \multicolumn{5}{|c|}{ Cantata a 2 per il SS. Natale (Delia, Dori) } \\
\hline & $\begin{array}{l}\text { Kantatenkopie } \\
\text { von Francesco } \\
\text { Lanciani, } \\
\text { 31.12.1714 }\end{array}$ & & unbekannt & $\begin{array}{l}\text { Die Kantatenkopie ist nicht } \\
\text { erhalten, Abrechnung: I-Rasv, } \\
\text { Fondo Ruspoli Marescotti. Filza } \\
\text { delle Giustificazioni del Libro } \\
\text { Mastro. Tomo II, B 58, } 1714 \text { (fasc. } \\
\text { 83). }\end{array}$ \\
\hline \multicolumn{5}{|c|}{ Cantata a 5 per il SS. Natale (3 Angeli, Pastore, Herode) } \\
\hline & $\begin{array}{l}\text { Kantatenkopie } \\
\text { von Francesco } \\
\text { Lanciani, } \\
\text { 20.12.1715 }\end{array}$ & & unbekannt & $\begin{array}{l}\text { Die Kantatenkopie ist nicht } \\
\text { erhalten, Abrechnung: I-Rasv, } \\
\text { Fondo Ruspoli Marescotti. Filza } \\
\text { delle Giustificazioni del Libro } \\
\text { Mastro. Tomo II, B 60, } 1715 \text { (fasc. } \\
\text { 118). }\end{array}$ \\
\hline
\end{tabular}

Andrea Zedler (andrea.zedler@ur.de), Lehrstuhl für Bayerische Landesgeschichte, Universität Regensburg.

Magdalena Boschung (magdalena.boschung@bluewin.ch), Institut für Musikwissenschaft, Universität Bern. 


\section{ABSTRACT \\ „PER L'ALLUSIONE ALLE CORRENTI COSE D'ITALIA“ ANTONIO CALDARA'S ROMAN CHRISTMAS CANTATAS FOR THE POPE AND THE PRINCE}

The present study examines the three preserved Christmas cantatas (Vaticini di pace, Vo' piangendo e sospirando and Amarilli vezzosa), which Antonio Caldara composed during his time in Rome between 1709 and 1716. Two of them were written per request of his patron, Principe Francesco Maria Ruspoli and one was composed for the Christmas festivities at the Pontifical court of Pope Clement XI. The study discusses the performance context of both the Apostolic Palace and the Ruspoli court. It also brings into perspective the problems which arose around the dating of the musical sources mentioned in this study. A text analysis of the works highlights references to contemporary political situations as well as dramaturgical and content-related connections between the texts. The analysis has shown, that the politically desired image of the Pope as a facilitator for peace in the War of the Spanish Succession plays a central role in Caldara's cantata for the Apostolic Palace, Vo' piangendo e sospirando (1713), as well as in Vaticini di pace (1712). An analysis of the music shows a correlation between Vo' piangendo e sospirando and Amarilli vezzosa (1713 and 1714 respectively), a cantata which has been preserved in two different versions. An intentional borrowing seems plausible, since the meaningful connection between the two cantatas shows Ruspoli's close relation and gratitude towards the Pontifical court, as well as his important social status as the patron of the Amarilli vezzosa.

\section{Key words}

Antonio Caldara, Christmas cantatas, Rome, Pope Clement XI., Francesco Maria Ruspoli

\section{Bibliography}

ADAMI, Andrea. Osservazioni per ben regolare il coro dei Cantori della Cappella pontificia. Rom: Antonio de Rossi, 1711.

Archivio della R. Società Romana di Storia Patria. Vol. XXIII, Fasc. I-II. 1900.

BOSCHUNG, Magdalena. Antonio Caldaras Serenata Il trionfo d'amore: Frankreichrezeption im Dienste adeliger Selbstdarstellung. In Die italienische Kantate im Kontext aristokratischer Musikpatronage, hrsg. von Berthold Over. Berlin: Merseburger (M.A.R.S. - Musik und Aristokratie im Rom des Sei-/Settecento, 3) (im Druck).

BOSCHUNG, Magdalena. Die Kantaten Antonio Caldaras für Principe Francesco Maria Ruspoli-Form und Funktion. Berlin: Merseburger, (M.A.R.S. - Musik und Aristokratie im Rom des Sei-/Settecento, 5) (in Vorbereitung).

CALDARA, Antonio - GINI, Paolo. Cantata da recitarsi la notte del SS ${ }^{\text {mo }}$ Natale nel Palazzo Apostolico l'anno MDCCXIII. Rom: Stamperia della Reverendissima Camera Apostolica. 1713, [cit. 2014-01-02]. URL: <http://daten.digitale-sammlungen.de/bsb00049909/image_1>.

CANCELLIERI, Francesco. Descrizione de Tre Pontificali che si celebrano nella Basilica Vaticana per le Feste di Natale di Pasqua e di S. Pietro. Rom: Stamperia Vaticana, 1788.

CASAREGI, Giovanni Bartolommeo (Eristo Faresio). E quando sia, che bella Pace amica. In Rime Degli Arcadi. Tomo Quinto. All'Illustriss. ed. Eccellentiss. Signore La Signora D. Maria Isabella Cesi Ruspoli Principessa di Cerveteri, \&., hrsg. von Giovanni Mario Crescimbeni. Rom: Antonio de Rossi, 1717, S. 262-266.

CRESCIMBENI, Giovanni Mario. L'Istoria della volgar Poesia scritta da Gio. Mario Crescimbeni. Canonico di Santa Maria in Cosmedin, e Custode d'Arcadia. Nella Seconda Impressione. Venedig: Lorenzo Basegio, 1731. 
CRESCIMBENI, Giovanni Mario. Storia dell'Accademia degli Arcadi istituita in Roma l'anno 1690. Rom 1712, Reprint, Bulmer: London, 1805.

DURCHHARDT, Heinz - ESPENHORST, Martin (Hrsg.). Utrecht - Rastatt-Baden 1712-1714. Ein europäisches Friedenswerk am Ende des Zeitalters Ludwig XIV. Göttingen: Vandenhoeck \& Ruprecht, 2013.

FRANCHI, Saverio. Il principe Ruspoli. L'oratorio in Arcadia. In Percorsi dell'oratorio romano. Da ,Historia Sacra “ a Melodramma Spirituale, hrsg. von dems. Rom: Ibimus, 2002, S. 245-316.

FRANCHI, Saverio. Mecenatismo musicale e poesia per musica a Roma nei primi decenni dell'Arcadia. In Atti e memorie dell'Arcadia, 2012, 1, S. 81-116.

GASPARINI, Francesco - BALDINI, Antonio. Cantata da recitarsi nel palazzo Apostolico la Notte del SS.mo Natale nel Palazzo Apostolico l'anno MDCCXVI. Rom: Stamperia della Reverenda Camera Apostolica, 1716. [cit. 2014-01-02]. URL: <http://books.google.com/books?vid=IBNR:CR000610965>

GIANTURCO, Carolyn.,Cantate Spirituali e Morali‘, with a Description of the Papal Sacred Cantata Tradition for Christmas 1676-1740. In Music \& Letters, 1992, 73/ 1, S. 1-31.

GINI, Paolo. Vaticini di Pace. Componimento per Musica. Da cantarsi nel Palazzo Apostolico. Rom: Luca Antonio Chracas, 1703 [cit. 2014-01-02]. URL: < http://www.mdz-nbn-resolving.de/ urn/resolver.pl?urn=urn:nbn:de:bvb:12-bsb10578629-1 >.

JOHNS, Christopher M. S. Papal Art and Cultural Politics: Rome in the Age of Clement XI. Cambridge: University of Cambridge Press, 1993.

KIRKENDALE, Ursula. Antonio Caldara. Life and Venetian-Roman Oratorios, 2. durchgesehene und übersetzte Aufl. Florenz: Leo S. Olschki Editore, 2007.

KIRKENDALE, Ursula. The Ruspoli documents on Handel. In Journal of the American Musicological Society, 1967, 20, S. 222-273.

KIRKENDALE, Ursula. The War of the Spanish Succession Reflected in Works of Antonio Caldara. In Acta Musicologica, 1964, 36/4, S. 221-233.

KIRKENDALE, Warren. Zu Handschriften von Händel und Caldara in der Santini-Sammlung. In Studi musicali, Nuova serie, 2013, IV/1, S. 61-76.

KÜPPER, Joachim. Petrarca. Das Schweigen der Veritas und die Worte des Dichters. Berlin, New York, N.Y.: de Gruyter \& Co, 2002.

LEONE, Rossella. Il Museo di Roma racconta la città. Roma: Gangemi, 2002.

LEONIO, Vincenzo. Perché il nascimento di Cristo Signor nostro si manifestasse prima d'ogni altro a' Pastori. Ragionamento del medesimo Uranio Tegeo fatto a' 4 di Gennaio 1713 nella Cancelleria Apostolica, Capanna dell'Acclamato Crateo Ericinio [Pietro Ottoboni], ove gli Arcadi sogliono ragunarsi ogni anno a celebrare la sollenità del Santiss. Natale, lor Tutelare. In Prose degli Arcadi, Vol. I, hrsg. von Giovanni Mario Crescimbeni. Rom: Antonio de Rossi, 1718, S. 363-383.

MARX, Hans Joachim. „... da cantarsi nel Palazzo Apostolico“. Römische Weihnachtsoratorien des 17. Jahrhunderts. In Musikkulturgeschichte. Festschrift für Constantin Floros zum 60. Geburtstag, hrsg. v. Peter Petersen. Wiesbaden: Breitkopf \& Härtel, 1990, S. 415-424.

MARX, Hans Joachim. Römische Weihnachtsoratorien aus der ersten Hälfte des 18. Jahrhunderts. In Archiv für Musikwissenschaft, 1992, 49/3, S. 163-199.

NAVACH, Lisa. , Cantando il duol dell'alme“. Analisi ed edizione critica delle cantate con violini di Francesco Gasparini, 2 Bd., Diss. masch, Università di Pavia, 2004.

NECCHI, Rosa. Marte nel Bosco Parrasio. La Rappresentazione della guerra nelle Rime degli Arcadi. In Per violate forme. Rappresentazioni e linguaggi della violenza nella letteratura italiana, hrsg. von Fabrizio Bondi und Nicola Catelli. Lucca: Maria Pacini Fazzi Editore, S. 47-60.

OVER, Berthold. Die Texte von Händels italienischen Kammerkantaten. In Händels Kirchenmusik und vokale Kammermusik (Das Händel-Handbuch 4), hrsg. von Michele Calella und HansJoachim Marx. Laaber: Laaber Verlag, 2012, S. 344-360.

PRESS, Volker. Kriege und Krisen. Deutschland 1600-1715. München: C.H. Beck, 1991. 
RIEPE, Juliane. Händel vor dem Fernrohr. Die Italienreise. Beeskow: ortus (Studien der Stiftung Händel-Haus 1), 2013.

SAMERSKI, Stefan. ,, Wie im Himmel, so auf Erden"? Selig- und Heiligsprechung in der Katholischen Kirche 1740 bis 1870. Stuttgart: Kohlhammer, 2002.

SCHNETTGER, Matthias. Der Spanische Erbfolgekrieg. 1701-1713/14. München: C. H. Beck, 2014.

TABACCHI, Stefano. L'impossibile neutralità. Il papato, Roma e lo Stato della Chiesa durante la Guerra di Successione spagnola. In Famiglie, nazioni e Monarchia. Il sistema europeo durante la Guerra di Successione spagnola, hrsg. von Antonio Álvarez-Ossorio Alvariño. Rom: Bulzoni, 2004, S. 223-244.

THEUER, Franz. Brennendes Land, Kuruzzenkriege. Ein historischer Bericht, Ein Böhlau-Sonderband. Wien: H. Böhlau, 1984.

ZEDLER, Andrea. Antonio Caldaras Kantatenschaffen zwischen römischen Conversazioni und dem Zeremoniell des Wiener Hofes. In Studien zur Musikwissenschaft, 2013, 57, S.117-140. 